\title{
Design, Fabrication, and Characterization of an Electrostatically Actuated Microfluidic Valve
}

\author{
A Thesis \\ Presented To \\ The Faculty of California Polytechnic State University \\ San Luis Obispo
}

In Partial Fulfillment

Of the Requirements for the Degree

Master of Science in Engineering with Specialization in Materials Engineering

By

Ryan D. Rivers

June 2010 
(C) 2009

Ryan D. Rivers

ALL RIGHTS RESERVED 


\section{COMMITTEE MEMBERSHIP}

Title:

Author:

Date Submitted:

Committee Chair:

Committee Member:

Committee Member:
Design, Fabrication, and Characterization of an Electrostatically Actuated Microfluidic Valve

Ryan D. Rivers

June 2010

Dr. Richard Savage

Professor of Materials Engineering

California Polytechnic State University, San Luis Obispo

Dr. Linda Vanasupa

Professor of Materials Engineering

California Polytechnic State University, San Luis Obispo

Dr. David Clague

Professor of Biomedical Engineering

California Polytechnic State University, San Luis Obispo 


\section{Abstract}

Design, Fabrication, and Characterization of an Electrostatically Actuated Microfluidic Valve

\section{Ryan D. Rivers}

Microfluidic device construction uses certain critical structures throughout many different applications. The valve structure remains one of the primary structures that present a barrier to miniaturization and portability. The extensive support devices required to power common microfluidic valves remove a significant amount of freedom from microfluidic device design. Moving to electrostatic methods of actuation could reduce the overall footprint of the microfluidic valve. This thesis covers three concept prototypes. Concept I presents an attempt at inlaying gold electrodes into polydimethylsiloxane substrates. Concept II attempts to use liquid silver injected into channels as electrode materials. Concept III uses aluminum sputtering to fabricate valve electrodes. Each device encountered complications during fabrication which led to improved fabrication guidelines for future devices. Designing and fabricating these concept devices required the development of several new processes in the clean room, including RIE Plasma bonding, PDMS sputtering techniques, and multilayer PDMS thin film fabrication. The PDMS sputtering technique in particular allows profilometry measurement of PDMS surfaces without risk of damaging the profilometer tip, a development that could allow for much more control over PDMS film thicknesses in future projects. 


\section{Acknowledgements}

First and foremost, thanks go to Dr. Richard Savage for his invaluable advice and support throughout this project. This project operated solely on Clean Room funding, and despite that Dr. Savage managed to procure every item I requested. Dr. Savage's uncanny ability to get our extremely temperamental aligner to function has saved this project more than once. None of the concept devices would have seen fabrication if not for his efforts.

Thanks to Hans Mayer for the introduction to soft lithography and providing me with a copy of many of the core research articles published on this topic. Hans' experience with pneumatic valves and other basic device structures helped shape the fundamental design of the electrostatic valve, and his assistance with my training in SU-8 resist lithography prevented dozens of fabrication complications and helped understand many others. Hans' work with the TCS and JerseyCote release layer formed a key component of my multilayer fabrication.

Thanks to Nic Vickers for helping me solve the polymer-plasma interactions and helping me find flaws in experiment concepts. Few people at Cal Poly understand the chemistry behind PDMS better than Nic.

Thanks to Brian Stahl for his availability as a general expert in the lab environment, and for assistance with initial modeling of electromechanical behavior.

Thanks to Sean Kaylor for helping me realize I reached too far too fast with concepts I and II. His comments about "Trying too hard for a home run" instead of working with more simple devices inspired the much more simplified design of concept III. Additionally, his work with SU-8 resist helped avoid many fabrication complications during concepts I and II.

Thanks to Christine Ghent for the incredibly quick turnaround on parts requests and order placement.

Thanks to Dylan Chesboro, Matt Lewis, and all of the clean room team for keeping the machines running smoothly.

Thanks to the entire Cal Poly Materials Engineering department for the constant support, funding, and assistance. There's no better group of people l've ever met.

Thanks to my family and friends for their constant support, Particularly during any of the many, many rants that occurred during my time working on this project. Thesis projects have a way of wearing down on one's sanity, and my friends and family constantly inspired me to keep slugging it out and finish. 


\section{Table of Contents}

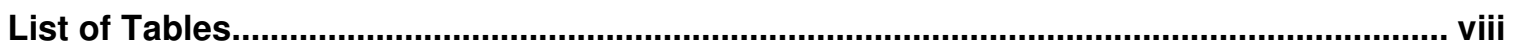

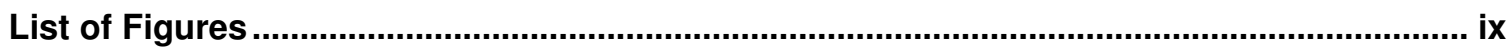

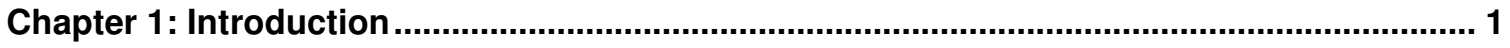

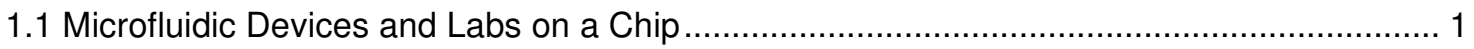

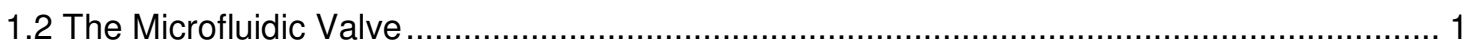

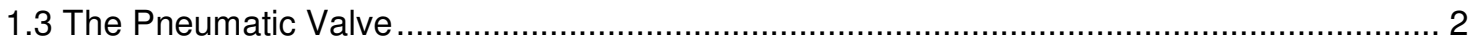

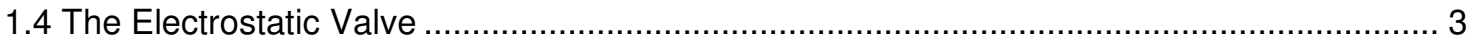

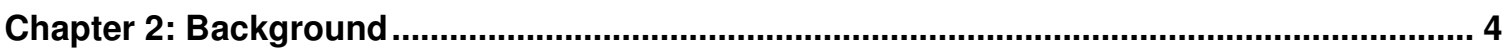

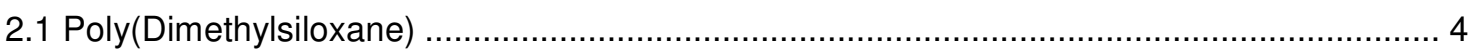

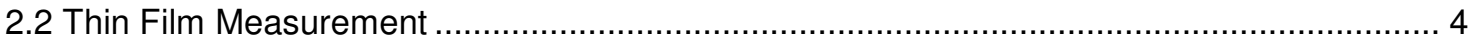

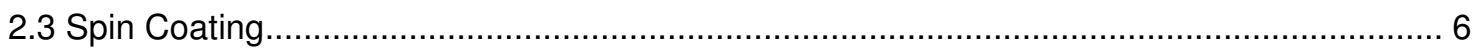

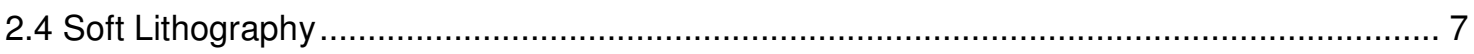

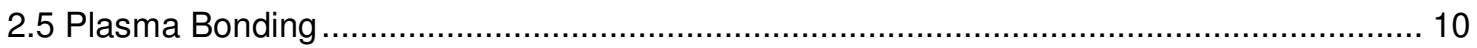

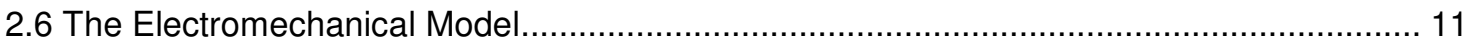

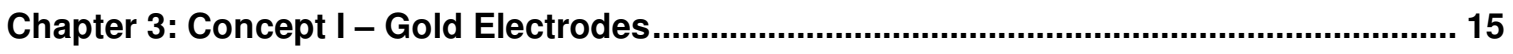

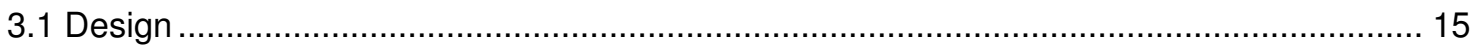

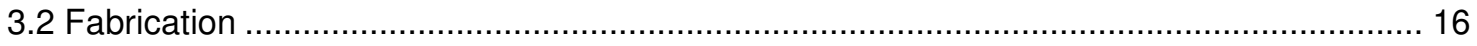

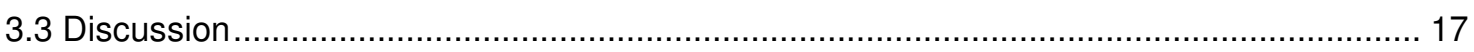

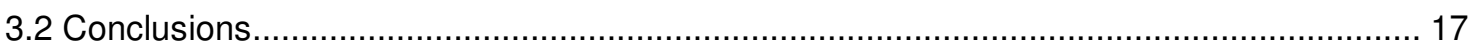

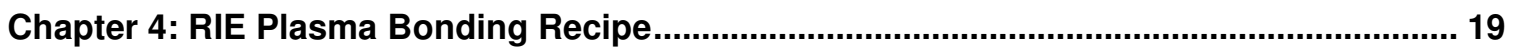

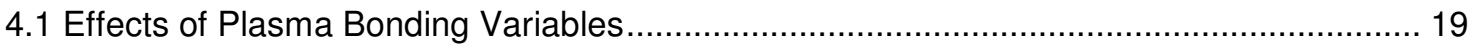

4.2 Experiment I - Exploration of Input Variables.............................................................. 19

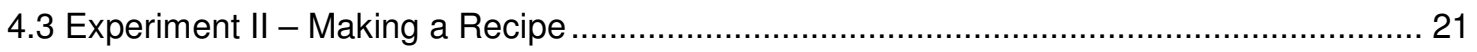

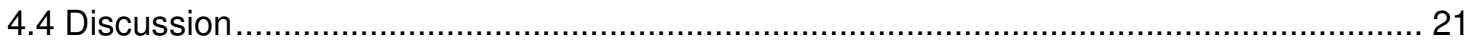

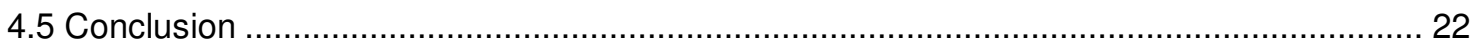

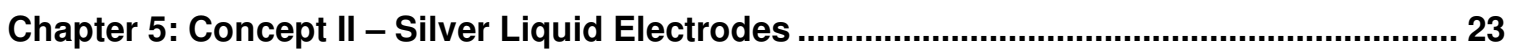




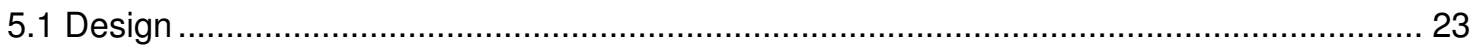

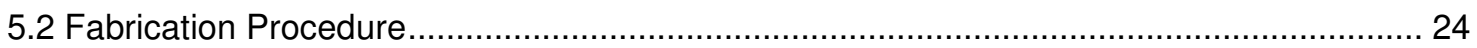

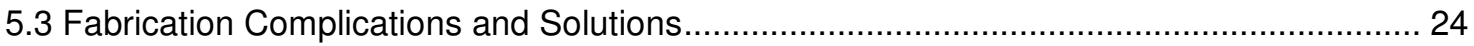

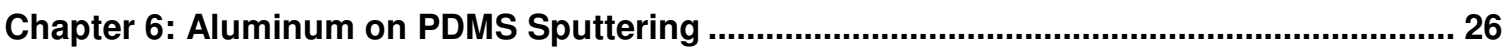

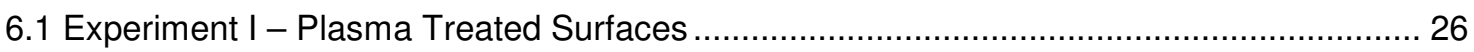

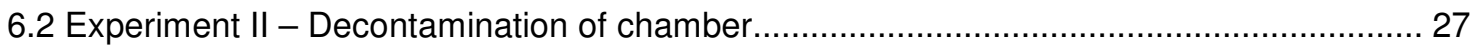

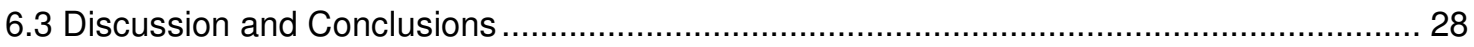

Chapter 7: Concept III - Aluminum Electrodes ................................................................. 29

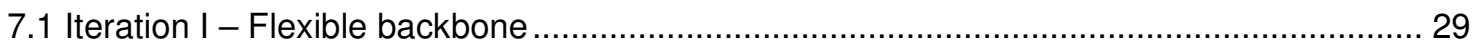

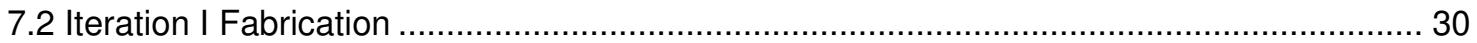

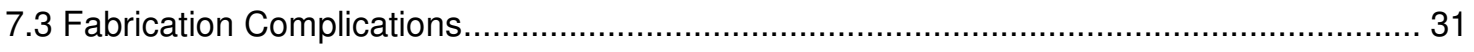

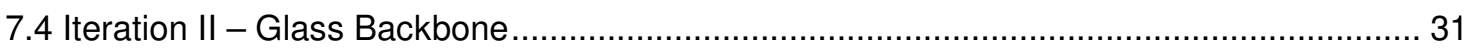

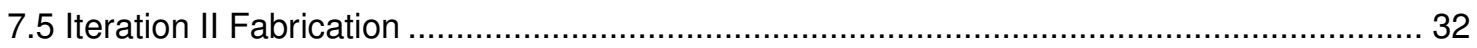

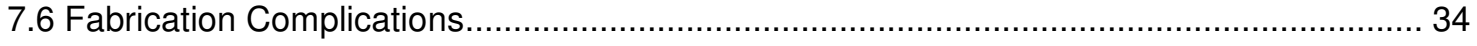

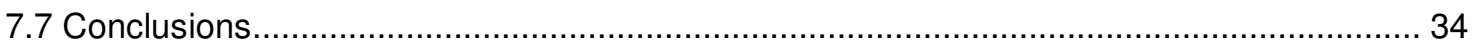

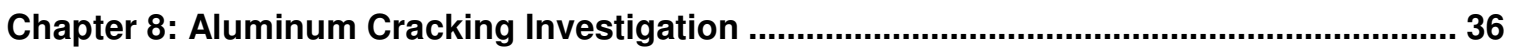

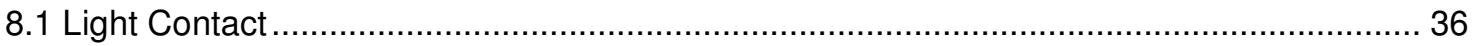

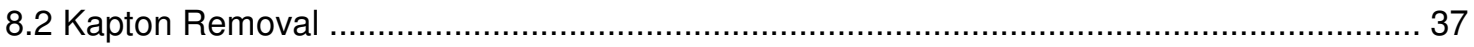

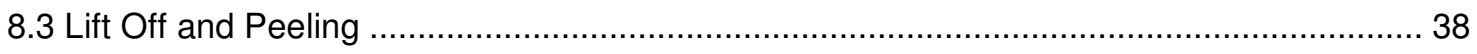

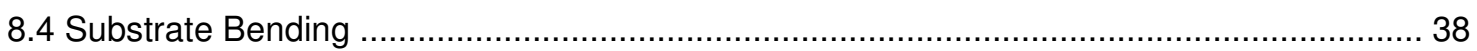

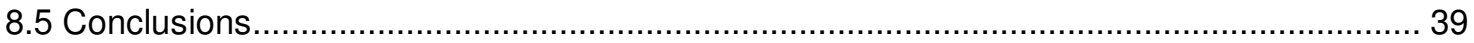

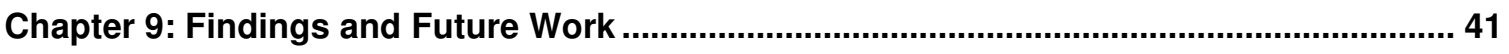

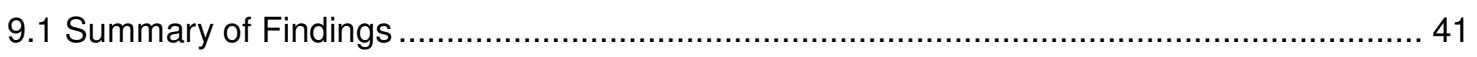

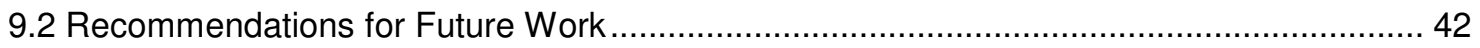

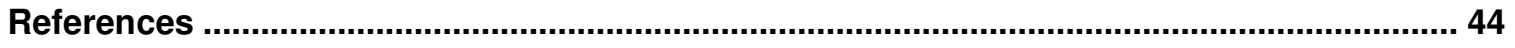

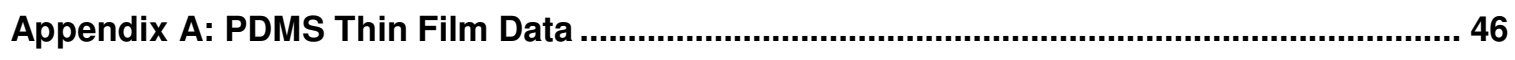

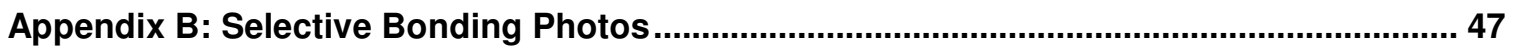




\section{List of Tables}

Table 4.1 Process Variable Inputs and Results for Experiment I ...................................... 19

Table 4.2 Time Inputs and Bond Quality Results of Experiment II....................................... 21 


\section{List of Figures}

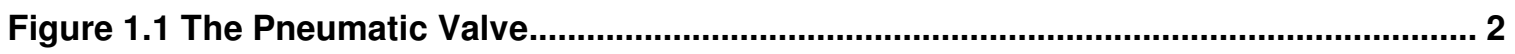

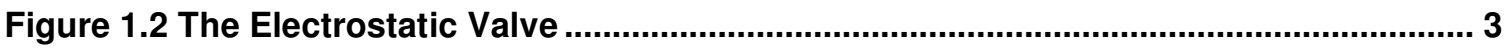

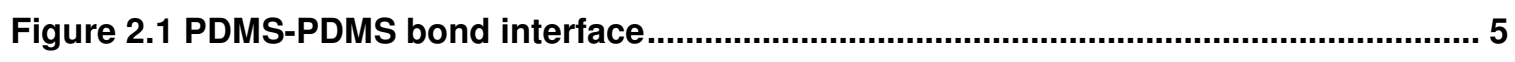

Figure 2.2 Diagram of Optical Error Induced by Sectioning …............................................. 5

Figure 2.3 Theoretical vs Empirical Spin Data ..................................................................... 7

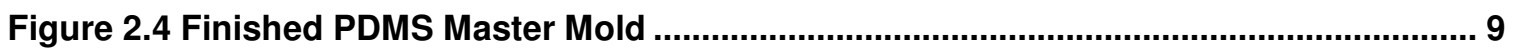

Figure 2.5 Comparison of Theoretical Geometric Effects on Actuation Voltage.................. 13

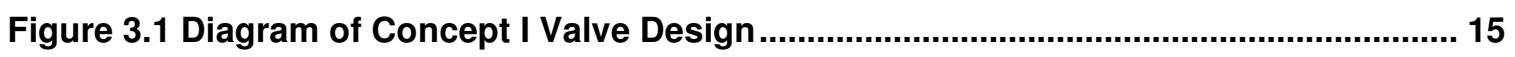

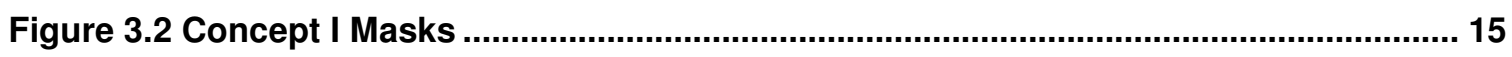

Figure 5.1 Diagram of Concept II Design and Masks.......................................................... 23

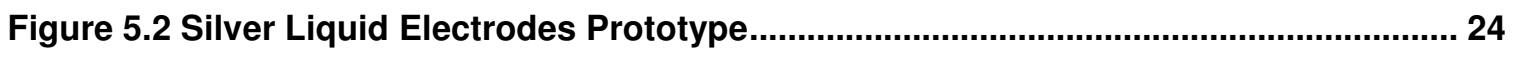

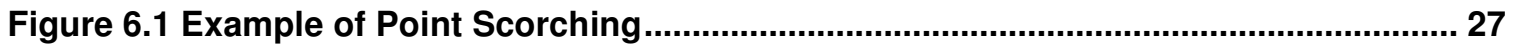

Figure 6.2 Comparison of Surface Scorching and Pristine Films ...................................... 28

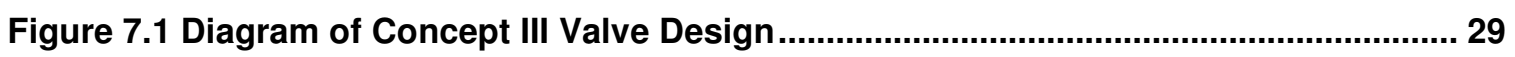

Figure 7.2 Stamp Block and Profilometer Scan Results ................................................ 32

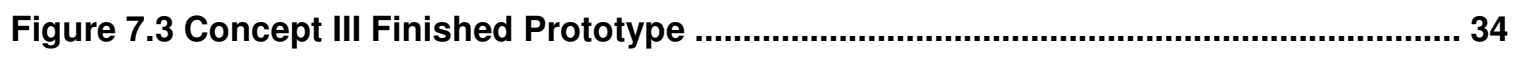

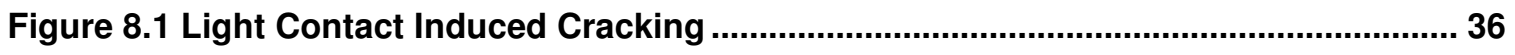

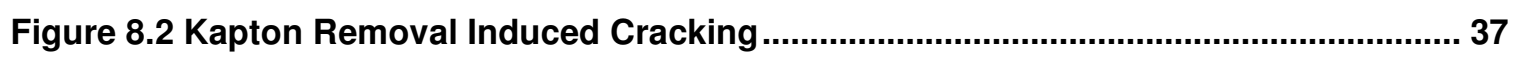




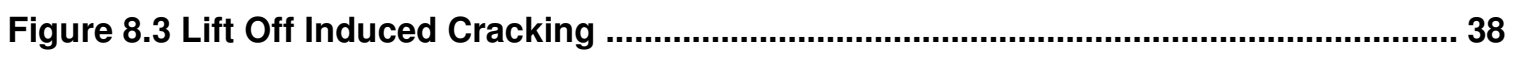

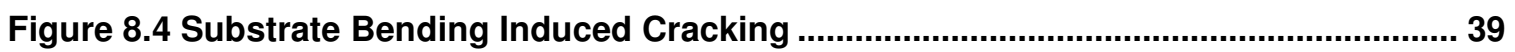

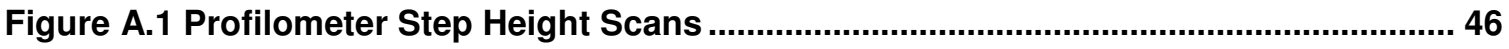

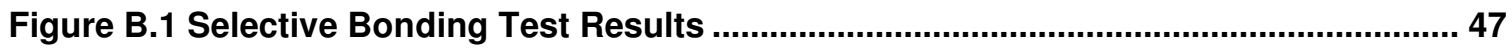

Figure B.2 Micrograph of Selective Bonding Interface........................................................ 47 


\section{Chapter 1: Introduction}

\subsection{Microfluidic Devices and Labs on a Chip}

Microfluidic systems attempt to significantly increase the efficiency and control of chemical and biological processes by shrinking reactions down to microscale dimensions. [1, 2] Microfluidic devices can mix chemicals, capture individual cells, perform electrophoresis, separate DNA, and in general perform many of the functions traditionally requiring complex lab setups. These microscale devices require far less space and support structure than their large scale counterparts. The active area of a microfluidic device usually spans less than $100 \mathrm{~mm} \times 100 \mathrm{~mm}$. A network of microfluidic channels, valves, mixers, pumps, and other structures within the active area accomplish the task of that particular device at a fraction of the size of a comparable laboratory system. [3-8]

This small size creates a unique opportunity for researchers. Fabricating a testing setup on a microfluidic platform theoretically allows for extremely portable and highly accurate testing devices. Such a lab-on-a-chip would allow on site biological and chemical testing and reduce the turnaround for test results significantly. [9]

\subsection{The Microfluidic Valve}

A microfluidic device consists of a series of channels that convey various fluids to different components of a microfluidic chip. Microfluidic valves direct flow through fluid channels in even the simplest devices, making them the most common form of microfluidic structure. Typical prototype device valves use simple pneumatic or hydraulic pressure to deflect a Poly(Dimethylsiloxane) (PDMS) membrane, closing a channel within various substrates. [10, 11, 12] The external power sources required to power pneumatic and hydraulic valves creates a barrier to lab-on-a-chip design. Large external pressure sources such as syringe pumps require large amounts of on-site power and storage space. A typical syringe pump weighs at least $2 \mathrm{~kg}$ and takes up at least twice as much space as the device it powers. Laboratory syringe pumps can require over $10 \mathrm{~W}$ of power to drive the syringe. [13] Each individually activated valve requires its own syringe pump, and each syringe pump requires a channel leading to the valves it actuates. These channels require a huge amount of space on a microfluidic device, and add at least one additional layer of channel structures to a device. These factors severely limit the complexity of a device using pneumatic systems.

The electrostatic valve can theoretically nullify these problems. A single electronic microchip can control hundreds of electrostatic valves in the same manner a computer CPU controls billions of transistors. While electrostatic valves require slightly higher voltages than syringe pumps, the 
capacitive nature of the circuits keeps current flow and thus power use to a minimum. The controller can also theoretically require a single power source with enough voltage to drive the electrostatic devices, making electrostatic valves a much more attractive choice for truly complex lab-on-a-chip devices. Electrical traces take up far less volume than pneumatic source channels and do not compromise the structural integrity of the device to the same degree. An electrostatic valve actually becomes more efficient with further miniaturization on the $Z$ axis. $A$ thinner channel's electrodes rest closer together, reducing the voltage necessary to actuate the device.

\subsection{The Pneumatic Valve}
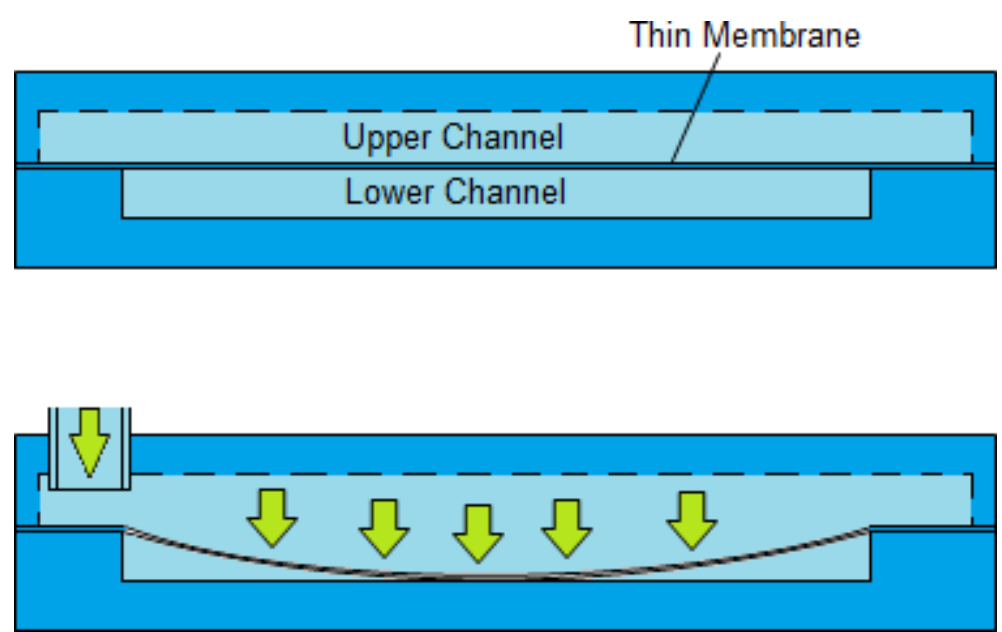

Figure 1.1 Above: A basic pneumatically actuated microfluidic valve design. Below: Behavior of thin membrane after pressurization of upper channel.

The key components of the pneumatic membrane valve most commonly used in microfluidic prototypes provide a starting point for modifying the valve design to use electrostatic actuation. [14] The typical pneumatic valve consists of a channel molded into a PDMS substrate and capped by a second, thin membrane of PDMS bonded to the open side of the channel. This assembly then bonds to a third layer containing a second channel which provides a conduit for pneumatic pressure. After completion, auxiliary devices pressurize the upper channel, deflecting the membrane until it squeezes the lower channel closed and prevents fluid flow. 


\subsection{The Electrostatic Valve}
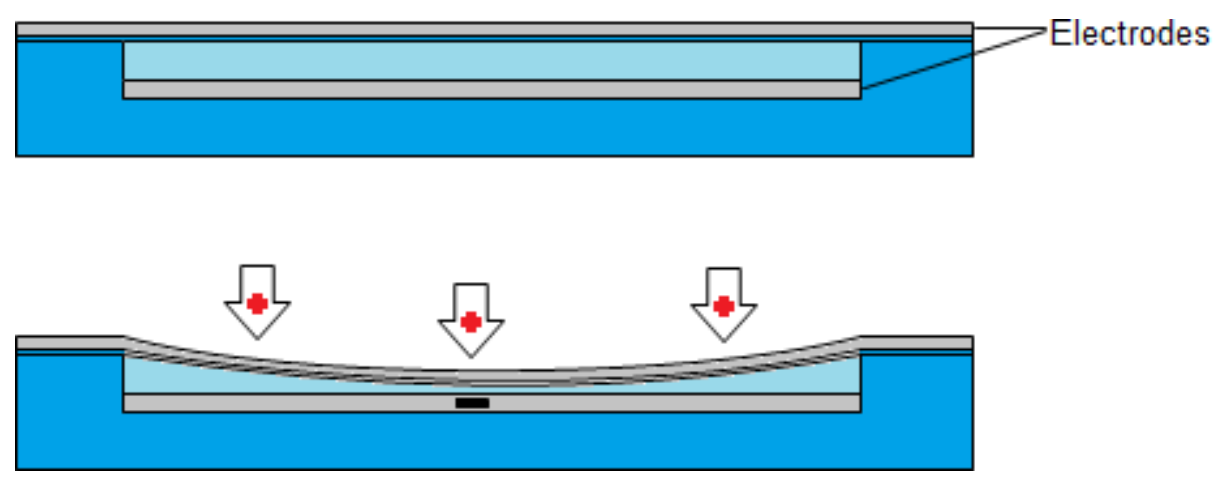

Figure 1.2 Above: A basic electrostatically actuated microfluidic valve design. Below: Behavior of thin membrane after application of driving voltage.

Theoretically, using electrostatically actuated valves in place of the traditional pneumatically actuated valve reduces the number and size of auxiliary devices required to power the device. Electrostatic force replaces pneumatic force in an electrostatic valve. Instead of an upper and lower channel separated by a thin membrane, two electrodes fabricated into the upper and lower walls of the channel become attracted by electrostatic forces generated by an applied voltage. The electrostatic attraction deflects the membrane and closes the fluid channel in a manner similar to the way the lower channel in a pneumatic valve closes.

The challenge in creating an electrostatic valve lies in fabrication. The question of how best to design not only the valve itself but the step by step process which creates it leads to several roadblocks. Metals typically do not bond to PDMS, making electrode fabrication extremely difficult.

Additionally, the ideal electrostatic valve maintains an extremely low aspect ratio. The thinner the channel, and the wider or longer the electrodes, the more force generated. However, too low an aspect ratio can lead to PDMS channel collapse which can destroy the device. 


\section{Chapter 2: Background}

\subsection{Poly(Dimethylsiloxane)}

Microfluidic systems research typically uses poly(dimethylsiloxane) (PDMS) as the primary substrate material of many prototype devices. These PDMS substrates possess many desirable qualities for use in microfluidic devices, including high flexibility, low chemical reactivity, high formability via soft lithography, and the ability to seal to other substrates via plasma bonding. [11] PDMS manufacturers ship the material in two parts: a base and a catalyst. Mixing the base and catalyst at a 10:1 ratio begins reactions that will crosslink the liquid polymer, eventually resulting in a solid elastomeric material. [15] Following the mixing, the curing PDMS requires vacuum treatment to remove air bubbles in the liquid. After removing the air the liquid PDMS can form into nearly any desired shape via casting. At room temperature, the curing reaction takes 1-2 days to complete. However, by elevating the temperature of the curing environment the process speeds up immensely. At $75^{\circ} \mathrm{C}$ the process requires less than 45 minutes, and at $130^{\circ} \mathrm{C}$ the process can take as little as 20 minutes. Higher temperatures during curing leave a small amount of stress in the PDMS proportional to the thermal contraction that occurs on cooling to room temperature.

Cured PDMS has a very low elastic modulus estimated at $750 \mathrm{kPa}$. [16] This low elastic modulus makes PDMS an ideal material for thin membrane valves. Very low amounts of pressure can close a PDMS membrane valve. The elastomeric nature of PDMS allows it to elastically stretch many times its equilibrium length before rupturing, stretching to cut off most or all of a channel. Most chemicals have no effect on fully cured PDMS. The nonpolar nature of PDMS gives it a hydrophobic surface and makes it impermeable to water. A cured substrate becomes all but impervious to chemical attack from anything other than organic solvents. Exposure to organic solvent solutions results in temporary swelling of the PDMS substrate as the solvent diffuses into the material. [17] The swelling subsides as the solvent evaporates and diffuses back out of the PDMS.

\subsection{Thin Film Measurement}

Thin film measurement presents one of the largest difficulties in designing a microfluidic device. Typical methods for measuring thin films such as profilometry and multiwavelength reflection spectroscopy cannot function with PDMS thin films. PDMS films rarely achieve thicknesses of less than $5 \mu \mathrm{m}$. The clean room's multiwavelength reflection spectroscopy system cannot analyze films that thick due to the bandwidth limitations of the light source and generally poor parameters for PDMS. [18] Profilometer tips stick to the extremely soft surface of PDMS, which can cause thousands of dollars in damage by breaking a profilometer tip as it travels across the surface of 
the PDMS. This leads to only one real thin film measurement option in cross sectioning and optical measurement.

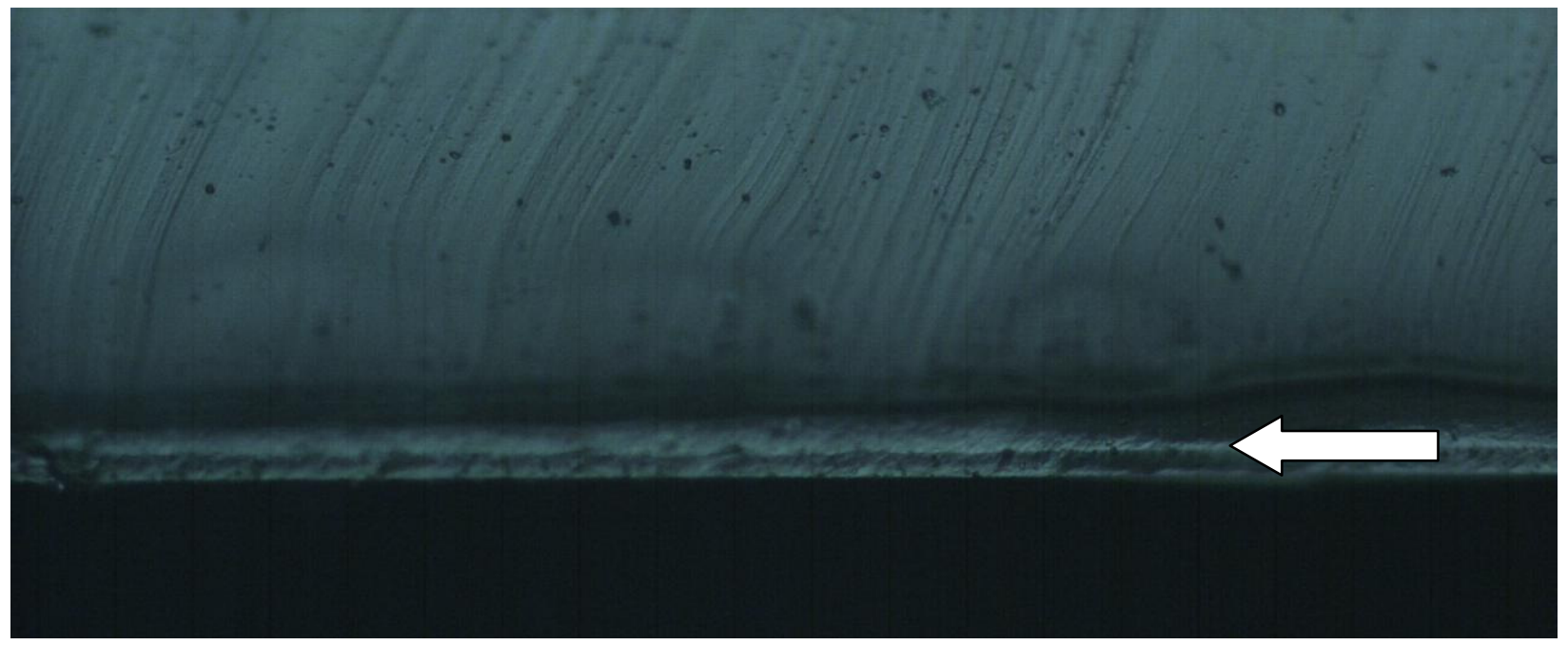

Figure 2.1 The bonding interface between the PDMS backbone and a $4 \mu \mathrm{m}$ "super-thin" film.

A taking a micrograph of a PDMS thin film's cross section reveals the thickness of the film by measuring the distance from the surface of the thin film to the interface between the film and the substrate bonded to it. Despite the ease of optical measurement, the technique retains several flaws. The interface between two PDMS blocks becomes inherently irregular after relaxing. This irregularity introduces a significant amount of error in thickness measurements.

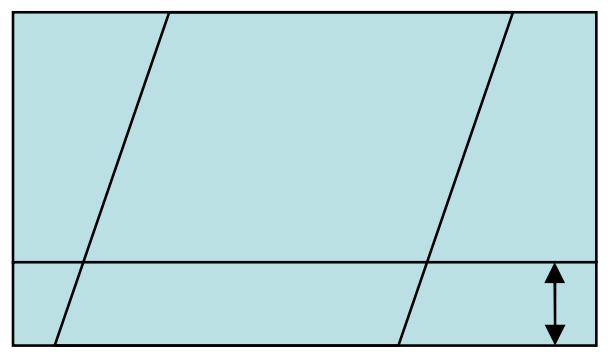

Actual Thickness

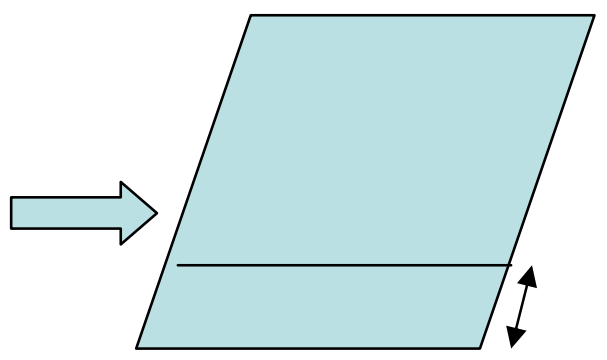

Measured Thickness

Figure 2.2 The act of sectioning a PDMS layer introduces a slight angle that can create error in measurement data.

Additionally, the process of sectioning these samples introduces a significant angle to the cross section relative to the microscope. Thicker films become increasingly difficult to measure accurately using the optical method, as the angle of cut can increase the apparent thickness of a film. 


\subsection{Spin Coating}

Most soft lithography techniques used during this project involved a process known as spin coating. A spin coating machine rotates a substrate at a sustained high angular velocity. The centripetal force spreads any liquid placed onto the spinning surface to a uniform thickness. Empirical thickness to spin speed curves exist for most commonly spun materials such as SU-8. PDMS has several different formulations, and no spin-speed curve data specifically for Sylgard 184 exists in any records at Cal Poly.

This leaves microdevice design with one final tool. Theoretical modeling of spin speed curves gives a basic idea of what to expect using a fluid of specific properties at a given spin speed for a nonvolatile, Newtonian fluid. [19]

$h=\left(\frac{3 \mu}{4 \rho}\right)^{\frac{1}{2}} \omega^{-1} t^{-\frac{1}{2}}$

The clean room uses Eq. 1 to model the behavior of fluids with unknown spin-speed properties. Eq. 1 describes the thickness $h$ in meters of a thin film of fluid with viscosity $\mu$ and density $\rho$ given the angular velocity in rotations per second $\omega$ and time $t$ of the spin process. Mixed PDMS has a viscosity of $4000 \mathrm{mPa} \cdot \mathrm{s}$, and a density of $1050 \mathrm{~kg} / \mathrm{m}^{3}$. [15] According to this model, spinning a film for 35 seconds at 1,500 RPM should create a $361 \mu \mathrm{m}$ thin film, spinning a film for 35 seconds at 2,000 RPM should create a $271 \mu \mathrm{m}$ thin film, and spinning a film for 120 seconds at 6100 RPM should create a $48 \mu \mathrm{m}$ thin film.

Qualitative inspection of any film spun under those conditions does not support this. A $400 \mu \mathrm{m}$ thick film should have far more structural integrity than the 1,500 RPM films exhibit. Any film spincoated above 1,000 RPM cures at a thickness that makes the film impossible to handle without first bonding some form of backbone or other supporting structure. 


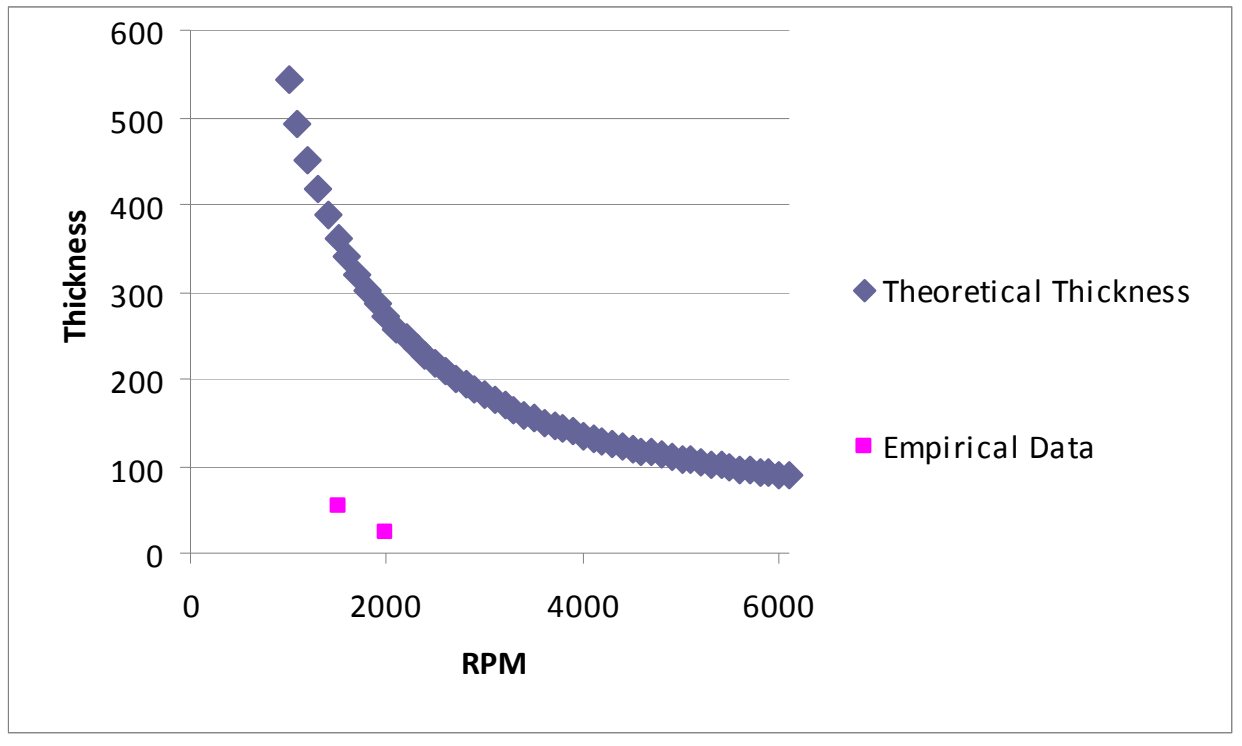

Figure 2.3 The few empirical data points obtained after the optimization of PDMS metallization indicate an extreme disparity between Eq. 1 and the actual thickness of PDMS.

Later quantitative inspection also fails to support this model. The 120 second, 6100 RPM super thin film shown above measures only $4 \mu \mathrm{m}$ optically. After the development of the metallization process described in Chapter 6, initial experimental data shows that a 35 second, 1,500 RPM spin resulted in a $53.5 \mu \mathrm{m}$ PDMS film, and a 35 second, 2,000 RPM spin speed generated a 23.5 $\mu \mathrm{m}$ film. Eq. 1 predicts numbers orders of magnitude off from this empirical data, which makes the use of Eq. 1 for PDMS thin films questionable.

Further investigation of the Sylgard 184 spin speed curve would assist greatly in the design phase of microdevices at Cal Poly. Clearly, the model does not accurately predict thin film thickness. Unfortunately too little time remained after the development of the metallization process to fully populate an empirical spin speed curve. The two points above currently make up the only profilometer-tested points on Cal Poly's PDMS spin speed curve.

\subsection{Soft Lithography}

PDMS substrates can inherit microscale features from master molds during the curing process.

This process, called soft lithography, allows for the fabrication of precise channel networks within a single PDMS layer. The primary limitation of soft lithography comes from its two dimensional nature. True three dimensional fabrication requires multiple molds and layers.

Soft lithography begins with the creation of a master mold. An SU-8 resist mold can provide extremely high precision in all dimensions. [10] Creating a master SU-8 mold begins by designing a mask in AutoCAD. A high resolution printout of emulsion onto a transparency sets the two 
dimensional pattern of each feature. At Cal Poly, typical masks with no features under $100 \mu \mathrm{m}$ use 5,000 DPI. Features under $15 \mu \mathrm{m}$ typically require 10,000 DPI.

After preparing the mask, a general fabrication procedure generates a master mold. Process variables such as spin speed and bake time remain dependant on the desired mold thickness. Exposure to water during the development creates an extremely thick white residue and effectively destroys the mold. The following values correspond to a typical $40 \mu \mathrm{m}$ tall mold. [20]

1. Clean a silicon wafer with a Piranha bath followed by a BOE bath

2. Dehydrate bake the wafer at $150 \stackrel{\circ}{\mathrm{C}}$ for 2 minutes

3. Transfer to spin coater

4. Pour a small amount of SU-8 2050 onto the wafer

5. Spin wafer at 4000 RPM for 30 seconds

6. Bake wafer at $65^{\circ} \mathrm{C}$ for 90 seconds

7. Bake at $95 \stackrel{\circ}{\circ}$ for 6 minutes

8. Transfer wafer to aligner and apply mask directly to wafer.

9. Cover wafer with 5 in $\times 5$ in glass plate and 5 in $\times 5$ in UV filter plate

10. Expose wafer to UV lamp for $140-180$ seconds

11. Bake wafer at $65^{\circ} \mathrm{C}$ for 1 minute

12. Bake at $95 \stackrel{\circ}{\mathrm{C}}$ for 6 minutes

13. Develop resist in SU-8 developer

14. Rinse with isopropyl alcohol

15. Hard bake at $130{ }^{\circ} \mathrm{C}$ for 2 minutes

16. Inspect mold for defects

A more advanced method of mold fabrication uses multiple SU-8 applications to fine tune the height. This technique allows creation of molds with features greater than $250 \mu \mathrm{m}$ tall, or features beyond the maximum height tolerances of SU-8 2007 and 2050. The process flow changes during the spincoating process. The following process creates a pattern greater than 250 microns tall.

1. Clean a silicon wafer with a Piranha bath followed by a BOE bath

2. Dehydrate bake the wafer at $150{ }^{\circ} \mathrm{C}$ for 2 minutes

3. Dehydrate bake wafer at $150 \stackrel{\circ}{C}$ for 2 minutes

4. Transfer to spin coater

5. Spin on SU-8 2050 at 1250 RPM for 30 seconds

6. Bake wafer at $65^{\circ} \mathrm{C}$ for 5 minutes

7. Bake at $95 \stackrel{\circ}{\circ}$ for 30 minutes

8. Repeat steps 20-22 varying spin speed and bake times as necessary

9. Transfer wafer to aligner and apply mask directly to wafer. 
10. Cover wafer with 5 in $x 5$ in glass plate and 5 in $x 5$ in UV filter plate

11. Expose wafer to UV lamp for 8 minutes

12. Bake wafer at $65^{\circ} \mathrm{C}$ for 5 minutes

13. Bake at $95 \stackrel{\circ}{\mathrm{C}}$ for 15 minutes

14. Develop resist in SU-8 developer

15. Rinse with isopropyl alcohol

16. Hard bake at $130 \stackrel{\circ}{ } \mathrm{C}$ for 2 minutes

17. Inspect mold for defects

Multilayer patterning techniques can also help bridge the gap between the low viscosity 2007 formulation and the high viscosity 2050 formulations of SU-8 which the clean room currently uses. Due to the low viscosity of SU-8 2007 obtaining features taller than 12-13 $\mu \mathrm{m}$ becomes difficult in one spin pass. However, stacking multiple layers of 2007 using the multilayer process can generate taller molds in 7-12 $\mu \mathrm{m}$ increments simply by varying the spin speed and soft bake times of each incremental layer. The SU-8 can expose as a single unit after application and soft baking of the individual layers.

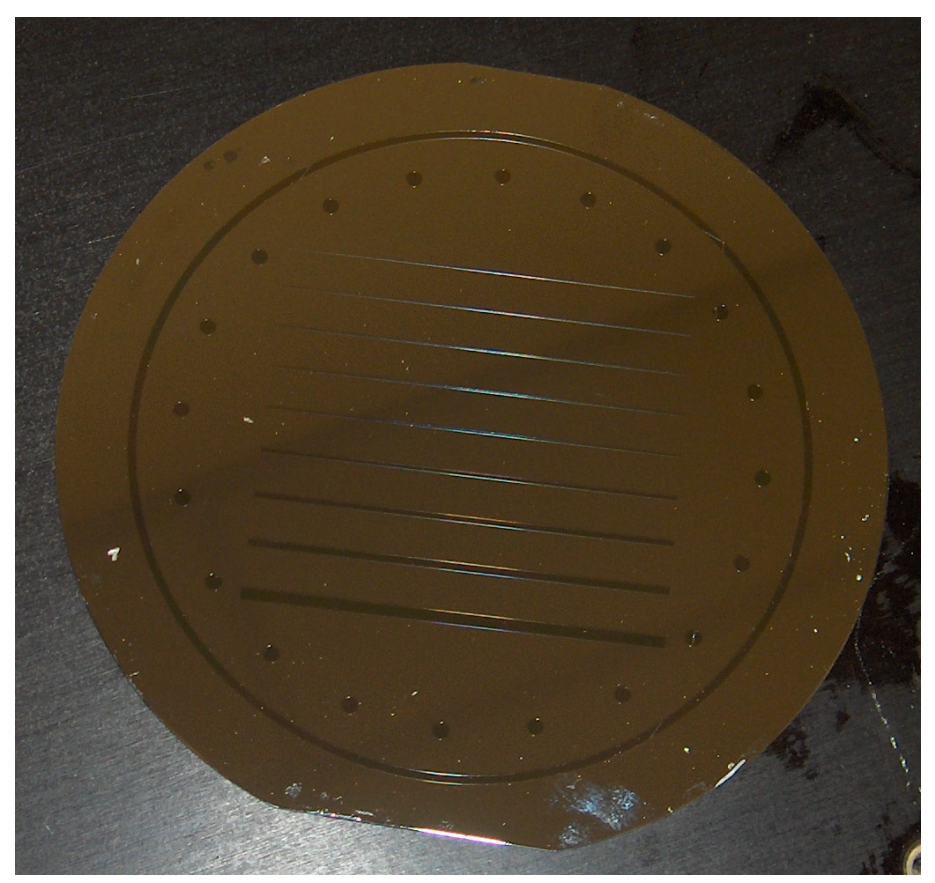

Figure 2.4 Finished PDMS master mold.

A finished master mold receives treatment with a release agent prior to use, ensuring easy lift off of thin films cast onto the mold. Without this release agent, removing PDMS cured in contact with the mold can require enough force to rupture and destroy the thin film. Vapor treatment with volatile compounds such as Trichlorosilanes (TCS) can create a self-assembled monolayer which acts as a release layer. A mix of ethyl alchohol and isopropanol called JerseyCote can also 
create a release layer when spun onto a wafer at 3000 RPM for 60 seconds. The Cal Poly clean room has effectively phased out TCS use in favor of the less dangerous and time consuming JerseyCote release agent.

A complete master mold can imprint multiple PDMS layers. Imprinting a layer requires spincoating liquid PDMS to the desired thickness over the mold, curing the PDMS, and lifting off the layer. Thin film layers typically need additional support during the lift off phase, using a backbone bonded to the film prior to removal from the mold.

\subsection{Plasma Bonding}

The key benefit to using PDMS as a substrate material lies in the ability of PDMS to bond to itself easily through relatively simple plasma treatments. The plasma bonding process creates a bond with a virtually undetectable interface which possesses material properties nearly identical to the bulk material. In essence, the surfaces of the two PDMS pieces cease to exist and a single macroscopically continuous block remains. [10]

This property has advantages when creating three dimensional structures. The ability to create a single object by stacking multiple layers on top of one another allows for complex, sealed channel networks on the micro and meso scale. These layers combine into an effectively continuous structure allowing remarkably complex designs with little to no leaks. The multilayer system also allows channels to cross other channels by routing over or under a channel. By manipulating these benefits, many features of piping systems can occur at the micro level.

PDMS can also bond to other substrate materials such as $\mathrm{SiO}_{2}$ and $\mathrm{Si}$ through the same method. Glass microscope slides make stable and relatively inexpensive device platforms for many devices. Typically these substrates see use in applications requiring a degree of rigidity.

To generate a plasma bond, PDMS substrates first need preparation. Each surface has tape attached and removed to clean the surface of any particulate matter such as dust or cloth fragments. Once cleaned, both substrates enter the vacuum chamber of a Rapid Ion Etching (RIE) machine. The RIE creates oxygen plasma in a low vacuum environment. Exposure to oxygen plasma replaces methyl groups on the surface of the substrate with an oxygen molecule. These oxygen molecules have dangling bonds. Upon venting the vacuum chamber, the hydrogen in the atmosphere bonds with the oxygen groups creating a large number of hydroxide groups on the surface of the substrate. The substrates require assembly within 10 minutes or the surfaces will relax too far to bond. Once assembled, the substrates bake within an oven at 65 to $75 \stackrel{\circ}{ } \mathrm{C}$ for at least 30 minutes. During this time, the hydroxide groups on the surfaces allow the surfaces to bond together via condensation, effectively removing the surface entirely. 
An argon plasma gun can also treat a PDMS surface. Plasma gun treatments require less lab space and less expensive equipment. However, using a plasma gun decreases control over exposure variables significantly, and can cause problems with sealing.

Underexposure or overexposure to plasma can thwart the bonding process by either failing to create enough hydroxide groups on the surface or causing too much damage to the surface to allow for bonding. In addition, PDMS begins to relax immediately after removing the treated surfaces from the plasma. The surfaces must come in contact and begin bonding within minutes of shutting off the oxygen plasma or the bond will fail. This severely restricts the alignment between layers.

Another concern during plasma bonding comes in the form of interchannel leaking. Channels placed too closely together in a layer may not have enough bonded area between them to hold back the pressures within the channel, generating ruptures and leaks. These cases generally occur due to poor or partial bonds. In some cases, a bond removed from an oven too early can fail to fully cure and rupture during operation as well. Channels closer than $5000 \mu \mathrm{m}$ separation can see fairly extreme interchannel leaking, especially with smaller channels which require more pressure to push the initial fluid/PDMS contact line fully through the device.

The most common form of bonding failure comes from channel collapse. Thin membranes covering channels can collapse during the bonding step and become permanently sealed to the floor of the channel. This phenomenon usually occurs only in channels with a greater than 10:1 width to depth ratio, though extremely wide channels greater than $500 \mu \mathrm{m}$ become more prone to collapse as the width increases.

Plasma treatment can also generate temporary hydrophilic surfaces. The hydroxide groups polarize the surface molecules and create a surface layer of hydrophilic material. Just as in plasma bonding, the surfaces relax over time to their original hydrophobic state.

\subsection{The Electromechanical Model}

Deriving a general model of the electromechanical behavior of an electrostatic valve provides a convenient design tool for determining the appropriate dimensions for a prototype valve. This electromechanical model incorporates geometric, mechanical, electrical, and material factors into a calculation resulting in the theoretical voltage required to fully close a valve of the given parameters.

The derivation begins with the relationship between an applied voltage across a parallel plate capacitor and its resulting force. [21]

$$
F=\frac{C}{2 d} V^{2}
$$


The applied voltage $V$ greatly affects the force output of an electrostatic device. The sum of the thickness of all components between the electrodes results in the distance $d$ between the plates.

$$
d=H+2 h
$$

Where $H$ describes the height of the channel, and $h$ describes the thickness of each wall membrane. The capacitance $C$ expands to:

$$
\begin{aligned}
& C_{\text {total }}=2 C_{\text {walls }}+C_{\text {fluid }} \\
& C_{\text {walls }}=\frac{\varepsilon_{0} \varepsilon_{\text {wall }} A}{h} \\
& C_{\text {fluid }}=\frac{\varepsilon_{0} \varepsilon_{\text {fluid }} A}{H}
\end{aligned}
$$

The relative permittivities $\varepsilon_{\text {fluid }}$ and $\varepsilon_{\text {wall }}$ describe the material contribution to the capacitance. Combining the area $A$ with the force applied $F$ results in a relationship between the applied voltage, channel geometry, and the pressure generated by the electrostatic attraction.

$$
P=\frac{F}{A}=\frac{\left(\frac{2 \varepsilon_{o} \varepsilon_{\text {walls }}}{h}+\frac{\varepsilon_{o} \varepsilon_{\text {fluid }}}{H}\right)}{2(H+2 h)} V^{2}
$$

Some rearrangement via algebra obtains the equation for the voltage required to apply a specific pressure to the valve membrane.

$$
V=\sqrt{\frac{2 P(H+2 h)}{\left(\frac{2 \varepsilon_{o} \varepsilon_{\text {walls }}}{h}+\frac{\varepsilon_{o} \varepsilon_{\text {fluid }}}{H}\right)}}
$$

Reasearch done by Kartalov et al. has constructed an empirical model for the pressure required to push down a rectangular valve membrane. [22]

$$
P=E \ln \left[1+\left(\frac{16 H^{2}}{3}\right)\left(\frac{1}{W^{2}}+\frac{1}{L^{2}}\right)+4 H\left(h^{3}+\frac{16 H^{3} h}{3}-\frac{16 H^{3}}{5}\right)\left(\frac{1}{W^{4}}+\frac{1}{L^{4}}\right)\right]
$$

Where $E$ represents the elastic modulus of the membrane material, $W$ represents the width of the membrane, and $L$ represents the length of the membrane. Using both Eq. 8 and 9 results in the theoretical voltage required to actuate a rectangular electrostatic valve of specific geometric and material construction. 
As an example, take a valve 750 microns wide and 750 microns long. Assume a 20 micron channel depth and 20 micron thickness in the upper and lower wall. A rough estimate of $750 \mathrm{kPa}$ accounts for the elastic modulus of the PDMS used in the construction of the valve. The final variables use water's relative permittivity of 80.1 for the fluid and 2.75 for the permittivity of the PDMS walls. A value of $5860 \mathrm{~Pa}$ for $P$ indicates the required force to close the valve. Generating this force requires $134 \mathrm{~V}$ across the electrodes.

The channel thickness, wall thickness, valve width, valve length, and fluid permittivity all change the behavior of the valve significantly. The fluid within the channel has the largest effect on actuation voltage. An extreme difference exists between the force generated through an active, water-filled channel and an empty, air-filled channel.
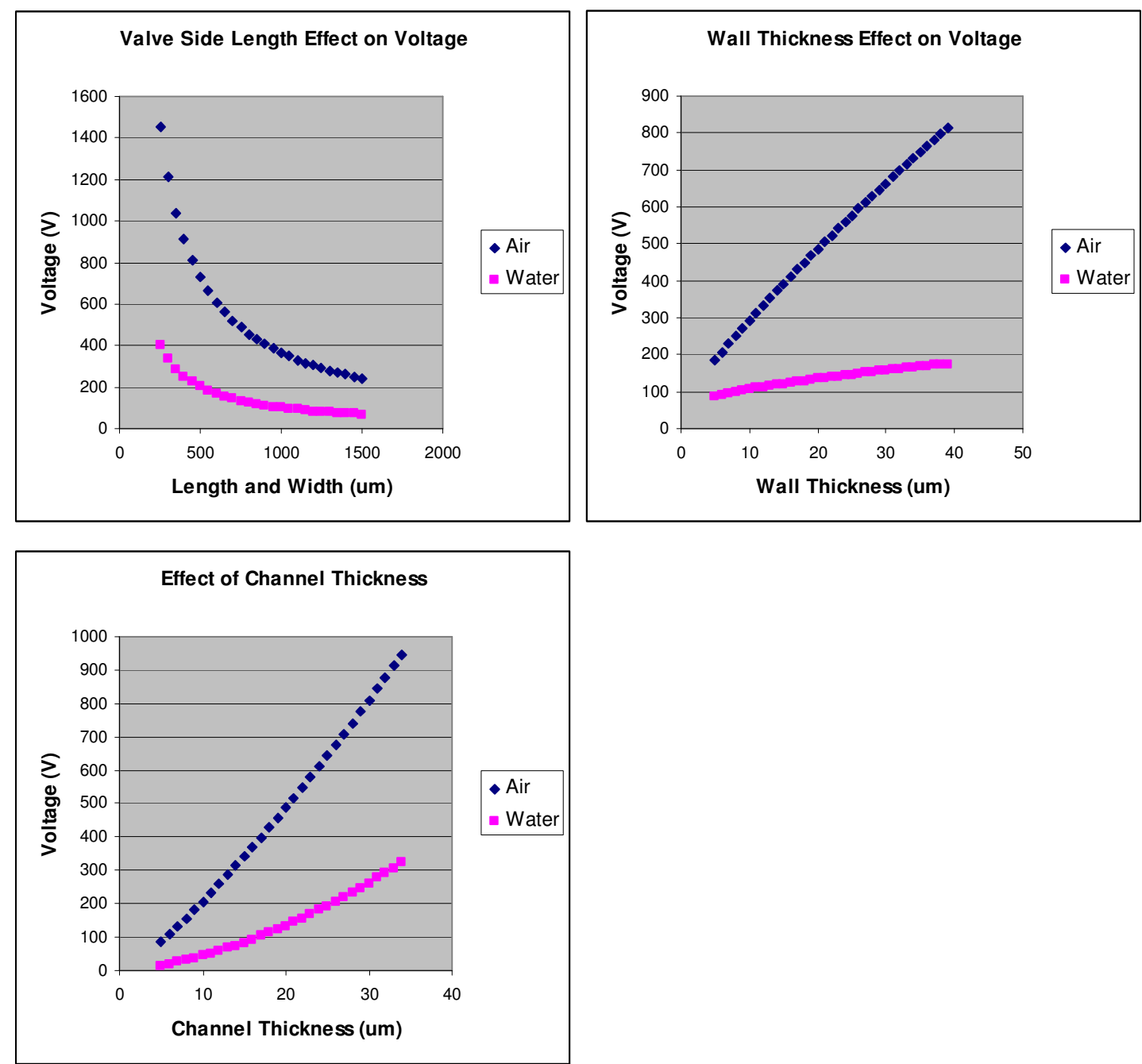

Figure 2.5 Each dataset assumes a value of $20 \mu \mathrm{m}$ for thicknesses and $\mathbf{7 5 0} \mu \mathrm{m}$ for valve side lengths not actively changing. The presence of water within the channel greatly decreases the actuation voltage regardless of valve geometry. 
Water's relative permittivity of 80.1 greatly amplifies the electrostatic field. By comparison the 2.75 relative permittivity of PDMS becomes completely overwhelmed in the presence of water. Specifically, the presence of water in the channel should allow much thicker walls between the electrodes and the channel itself. Increasing the thickness of the channel greatly increases the voltage required to actuate the device. The model also predicts severely increasing voltages for valves widths and lengths under $500 \mu \mathrm{m}$. 


\section{Chapter 3: Concept I - Gold Electrodes}

\subsection{Design}

The first concept design of the electrostatic valve uses PDMS as the primary substrate for all layers. A literature search found research detailing the successful transfer of gold patterns into PDMS. [22] Combining the transfer method with the PDMS plasma bonding process allows creation of a four layer electrostatic valve.

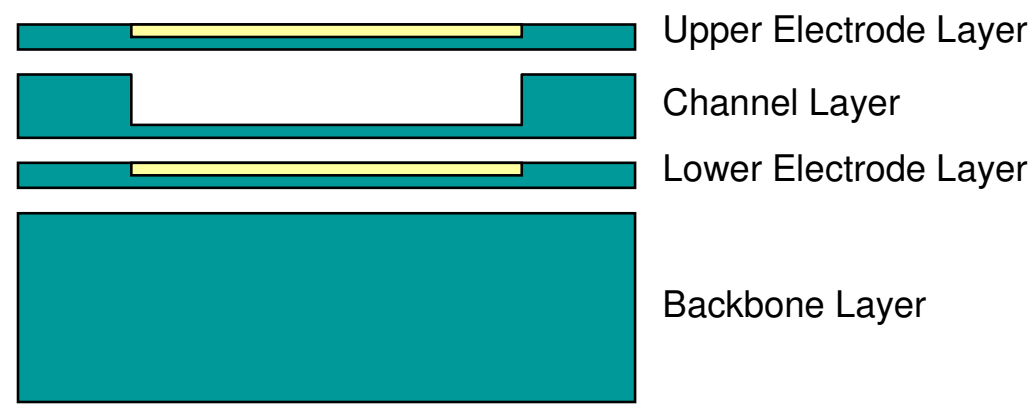

Figure 3.1 Breakdown of Concept I layer stack.

A $0.4 \mathrm{~cm}$ thick PDMS block forms the backbone layer. The backbone layer functions as a handle during the lift off phase of soft lithography, and as a stable platform to allow rough handling of the more sensitive layers during lab processes.
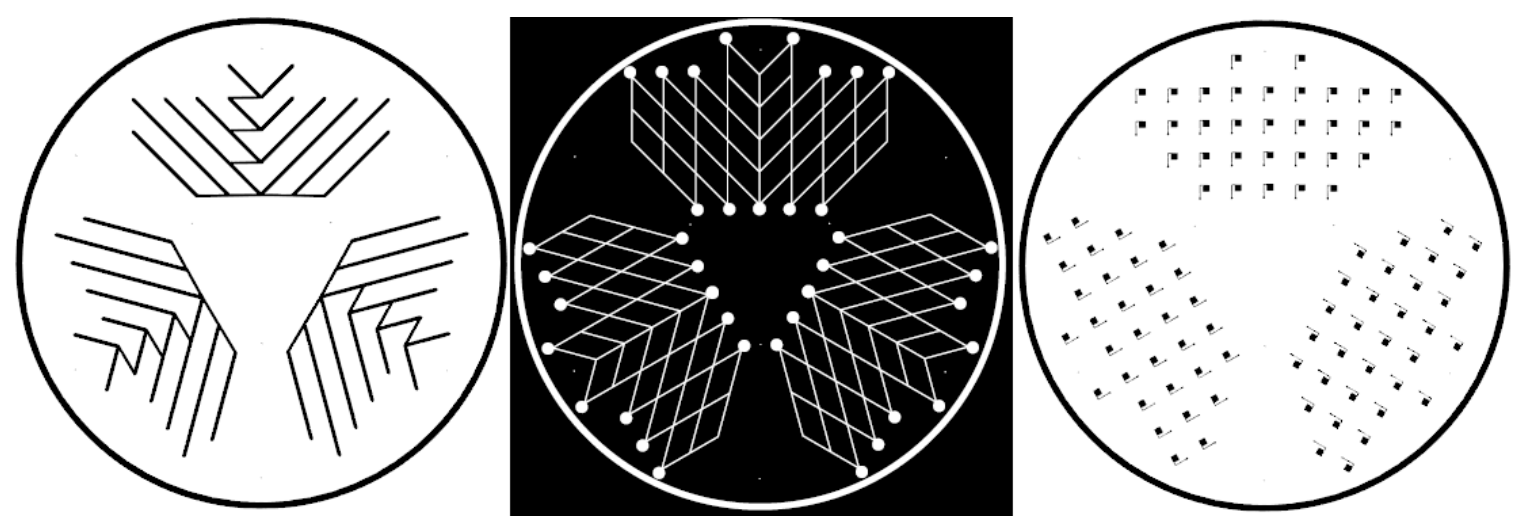

Figure 3.2 Left: Lower Electrode Layer gold pattern. Center: Channel Layer SU-8 pattern. Right: Upper Electrode Layer gold pattern.

The channel layer consists of channel structures imprinted into a PDMS thin film via soft lithography. The master mold for the channel layer contains three channel blocks. Each channel measures $100 \mu \mathrm{m}$ across and $15 \mu \mathrm{m}$ deep, while the Input ports measure $2 \mathrm{~mm}$ across. A $20 \mu \mathrm{m}$ PDMS thin film cast over these features leaves a $5 \mu \mathrm{m}$ membrane between the channel layer and the gold on the lower electrode layer. 
Both electrode layers contain $60 \mathrm{~nm}$ thick gold electrodes transferred into PDMS thin films. A vapor treatment of 3-Mercaptopropyl Trimethoxysilane (MPT) over the gold surfaces creates a self-assembled monolayer. This methoxy groups in MPT bond to the PDMS as it cures over the monolayer, creating an adhesion layer between the gold and the $20 \mu \mathrm{m}$ PDMS thin film, which improves the quality of the transfer. The lower electrode design allows a single point of electrical contact to ground the entire channel block.

Each prototype device contains 32 separate valves to ensure at least a few valves survive the various fabrication steps. A network of fluid channels connects these valves, designed to require a minimal amount input and output ports on the channel layer to reduce the amount of damage to the upper electrode layer during testing.

\subsection{Fabrication}

\section{Backbone Layer Fabrication Recipe}

1. Mix $35 \mathrm{ml}$ Sylgard 184 at a $10: 1$ base to catalyst ratio

2. Place mixture into vacuum chamber and degas for 10 minutes

3. Pour liquid PDMS into 6" petri dish

4. Cure PDMS in oven at $75^{\circ} \mathrm{C}$ for 45 minutes

\section{Upper/Lower Electrode Layer Fabrication Recipe}

1. Sputter gold onto wafer at $9 \mathrm{mTorr}$ and $60 \mathrm{~W}$ for 1 minute

2. Spin on HMDS primer at 500 RPM for 30 seconds

3. Spin on positive photoresist at $3000 \mathrm{RPM}$ for 30 seconds

4. Soft bake positive photoresist at $90 \stackrel{\circ}{\mathrm{C}}$ for 1 minute

5. Expose positive photoresist for 16 seconds

6. Develop positive photoresist using CD-26

7. Etch gold using $\mathrm{KI}$ based gold etchant

8. Vapor treat surface with MPT

9. Vapor treat surface with TCS

10. Spin on $3 \mathrm{ml}$ Sylgard 184 at 3000 RPM for 30 seconds

11. Cure PDMS in oven at $75 \stackrel{\circ}{\circ}$ for 45 minutes

\section{Channel Layer Fabrication Recipe}

1. Vapor treat channel layer master mold with TCS

2. Spin on $3 \mathrm{ml}$ Sylgard 184 at 2000 RPM for 30 seconds

3. Cure PDMS in oven at $75 \stackrel{\circ}{\mathrm{C}}$ for 45 minutes 


\subsection{Discussion}

Unfortunately, none of the devices ever fully survived the fabrication process. Multiple complications arose during fabrication of the active layers.

Initial designs left the electrode layer PDMS thin films so thin they fell apart during bonding and lift off. A decrease in spin speed to match the channel layer thickness solved this issue. In addition, combining the backbone and lower electrode layer reinforced the lower electrode layer during lift off.

The MPT adhesion layer failed to function properly. Two possibilities explain this phenomenon. First, the TCS release agent and the MPT adhesion agent could interfere with one another. Both the chemicals use the formation of a self-assembled monolayer via vapor treatment to change the properties of the surface. The TCS and MPT directly conflict with one another for monolayer formation. Second, the MPT could have failed to properly coat the gold traces.

Rearranging the process steps to expose the wafer to the TCS release agent prior to gold sputtering resulted in PDMS tearing during release of the layer. Additionally, the reorganized recipe continued to fail to fully transfer the gold films. Testing gold transfer with and without the MPT confirmed the MPT had no effect on transfer quality. This suggested the MPT vapor treatments failed to successfully coat the gold traces. MPT appears to require a vacuum assistance to properly vaporize and create a SAM on the wafer. The clean room has no vacuum chambers inside fume hoods, and the toxic nature of MPT precludes its use outside of a fume hood.

A thin oxide coating on surface of the wafer substrate succeeded in releasing the gold from the substrate without MPT adhesion layers. However, the gold thin film experienced severe delamination problems during wet etch processes.

Finally, the complexity of the design worked against success. While multiple valves existed on each block, the large blocks developed several leaks due to the uneven treatment from the plasma gun. While one or two electrodes might survive a transfer intact, no corresponding electrode ever survived to allow creation of a fully functional valve.

\subsection{Conclusions}

Concept I ruled out gold as an electrode material. The results of K.J. Lee et al. proved impossible to reproduce with the equipment available. The cost of electronics grade gold precluded further attempts at developing a method for full transfer. 
Concept I also revealed that extremely thin $(<10 \mu \mathrm{m})$ PDMS layers do not possess enough durability to reliably survive the lift off process. Additionally, concept I revealed the ineffectiveness of complex device designs. 


\section{Chapter 4: RIE Plasma Bonding Recipe}

\subsection{Effects of Plasma Bonding Variables}

Concept I shows the argon plasma gun's failure to adequately treat PDMS surfaces evenly. The AGS Plasma Systems RIE system allows more uniform exposure to plasma, and provides better control over relevant input variables. Time of exposure, chamber pressure, and RF power input all impact the quality of the PDMS bond. The plasma bonding process depends heavily on the amount of time the PDMS surface remains exposed to active plasma. Too short a duration fails to activate the surface. Too long a duration can result in an overdose of energy, which also leads to a failed bond. Chamber pressure affects the range of time in which a desirable bond will occur by modifying the amount of ions striking the surface of the material per second, while changing the RF power input modifies the energy with which each ion strikes the surface.

\subsection{Experiment I - Exploration of Input Variables}

The initial experiment helped glean information about how each of the three input variables affected bond quality. Previous experience with manipulating the plasma bonding parameters showed that time of exposure greatly affected bond quality results. Previous recipes had achieved bonding at both high pressures and low pressures.

Table 4.1 Process Variable Inputs and Results for Experiment I

\begin{tabular}{|l|l|l|l|l|}
\hline Run & Power (W) & Pressure $(\mathrm{mT})$ & Time $(\mathrm{sec})$ & Bond Quality \\
\hline A1 & 20 & 58 & 3 & Poor Bond \\
\hline A2 & 20 & 58 & 2 & Partial Bond \\
\hline A3 & 20 & 58 & 1 & No Bond \\
\hline B1 & 20 & 108 & 5 & Good Bond \\
\hline B2 & 20 & 108 & 3 & Partial Bond \\
\hline B3 & 20 & 108 & 1 & No Bond \\
\hline C1 & 50 & 108 & 5 & Partial Bond \\
\hline C2 & 50 & 108 & 3 & Partial Bond \\
\hline C3 & 50 & 108 & 1 & Good Bond \\
\hline D1 & 20 & 308 & 50 & No Bond \\
\hline D2 & 20 & 308 & 40 & Partial Bond \\
\hline D3 & 20 & 308 & 30 & Good Bond \\
\hline
\end{tabular}


The experiment helped determine the effect of changes to pressure and power input on the time required to create a strong and uniform bond. Each test involved two blocks of thermally cured PDMS treated under specific pressure, power, and time conditions.

The experiment tested each of four different power and pressure combinations. Cal Poly does not posess the necessary equipment to effectively test the strength of the bond. In lieu of quantitative data, qualitative testing must suffice. Three criteria describe a "good bond":

1. A complete bond, with no areas near edges which failed to bond.

2. The bond must withstand tearing across the interface, indicating the toughness of the bonded region to match or exceed the toughness of the bulk material.

3. The torn surface must give no visual indicator of where the interface between the two previous surfaces exists.

A "partial bond" complies with conditions two and three, but has areas where the bond failed to take hold. Typically, these areas occur near edges of the two PDMS substrates. Partial bonding can result from a lack or excess of plasma exposure. More extreme partial bonding issues occur from dirt or other contamination present on the substrates during plasma treatment. Devices which partially bond leak, causing cross channel contamination, and cannot properly function as microfluidic devices.

"No bond" means a test in which no bond occurred at all. Both pieces separated from each other without resistance. A no bond result indicates configurations that have too little or too much time of exposure, and as a result have failed to activate the surface of the substrate.

The results of runs $\mathrm{A} 1$ through $\mathrm{A} 3$ at $20 \mathrm{~W}$ and $58 \mathrm{mT}$ showed a lack of bonding at $1 \mathrm{sec}$ and a poor bond at $3 \mathrm{sec}$ of exposure time. However, at $2 \mathrm{sec}$ of exposure, the bond held strongly at most of the interface, though the edges failed to bond in many places.

Runs B1 through B3 increased the pressure to $108 \mathrm{mT}$ show a dramatic expansion of the window in time of exposure values in which a strong bond can occur. A $3 \mathrm{sec}$ treatment produced similar results to run $A 2$, while run $B 3$ produced a good bond with a $5 \mathrm{sec}$ treatment.

Runs C1 through C3 increased the power output to $50 \mathrm{~W}$ at $108 \mathrm{mT}$. The degradation in bond quality indicates that increasing the power input may cause general bond degradation.

Runs D1 through D3 returned the power output to $20 \mathrm{~W}$ at $308 \mathrm{mT}$. Previous recipes achieved successful bonds at timestamps greater than 30 seconds. The success of the 30 second sample's bond shows a much larger time window at the higher chamber pressure. 


\subsection{Experiment II - Making a Recipe}

The second experiment used the information gleaned from experiment I to explore the time of exposure window more effectively. All runs of this experiment took place at $20 \mathrm{~W}$ and $308 \mathrm{mT}$. The previous experiment's results indicated a lower power and higher pressure would extended the time of exposure window to an interval in which more samples could better indicate which times created optimal bonds.

Table 4.2 Time Inputs and Bond Quality Results of Experiment II

\begin{tabular}{|l|l|l|l|}
\hline Time (s) & Sample 1 & Sample 2 & Sample 3 \\
\hline 15 & Good Bond & Good Bond & Good Bond \\
\hline 20 & Good Bond & Good Bond & Good Bond \\
\hline 25 & Partial Bond & Partial Bond & Partial Bond \\
\hline 30 & Partial Bond & Partial Bond & Partial Bond \\
\hline 35 & Good Bond & Good Bond & Good Bond \\
\hline 40 & Partial Bond & Partial Bond & Partial Bond \\
\hline 60 & Partial Bond & Partial Bond & Partial Bond \\
\hline
\end{tabular}

Bonding results follow the same guidelines as those in experiment I. However, in experiment II, each surface remained covered by scotch tape until placed into the chamber by tongs. Thus, none of the partial bond results come from dirty surfaces.

The results seem to indicate a time interval of $15-20 \mathrm{sec}$ results in a good bond. This interval allows for much more human error than the previous interval of 2-3 seconds. The results also indicate good bonding at $35 \mathrm{sec}$ of exposure, however $30 \mathrm{sec}$ and $40 \mathrm{sec}$ of exposure both produce partial bonds, making $35 \mathrm{sec}$ runs a more difficult process to consistently execute.

\subsection{Discussion}

Overall, the results of the experiments indicate time as the most crucial variable in the process. Too much or too little time of exposure causes bond failure. Increasing pressure seems to increase the range of exposure times in which a good bond remains possible. Input power seems to have little to no effect on the time window. Increasing input power output appears to generate lower quality bonding results. The lower quality bonds could result from surface damage incurred by the higher energy ion impacts at higher power levels.

From an operator's viewpoint, the ideal recipe extends the time of exposure window to the point that an error of 1-2 sec in the process time does not compromise the bond's integrity. By increasing the pressure from $58 \mathrm{mT}$ to $308 \mathrm{mT}$, the time of exposure window increases 
significantly. Attempting to end the process within a $5 \mathrm{sec}$ window better allows for human error than a $1 \mathrm{sec}$ window. The larger $15-20 \mathrm{sec}$ time of exposure also allows the operator to check the chamber viewport to ensure the plasma struck successfully and confirm the sensitive power settings remain undisturbed.

\subsection{Conclusion}

A new plasma bonding recipe of $20 \mathrm{~W}, 308 \mathrm{mT}$, and $15 \mathrm{sec}$ reliably creates good bonds. The higher pressure allows for a much longer exposure window and suits a one-shot process with a high degree of human error. Choosing $15 \mathrm{sec}$ allows the operator to overshoot the time by 1-2 seconds and remain well within the parameters necessary for a consistently acceptable bond. 


\section{Chapter 5: Concept II - Silver Liquid Electrodes}

\subsection{Design}

The failure of the gold electrode transfer required a redesign of the electrode fabrication method. Rather than attempt to transfer metal into a PDMS layer, this iteration attempted to fill channels above and below the device with a conductive silver colloidal liquid commonly used to fix damaged traces on electronic equipment. Theoretically, where solid state conductors cracked and otherwise failed, the liquid conductor could self-heal. In addition, the fabrication process for the PDMS components used only known techniques in an effort to avoid unforeseen complications during fabrication.
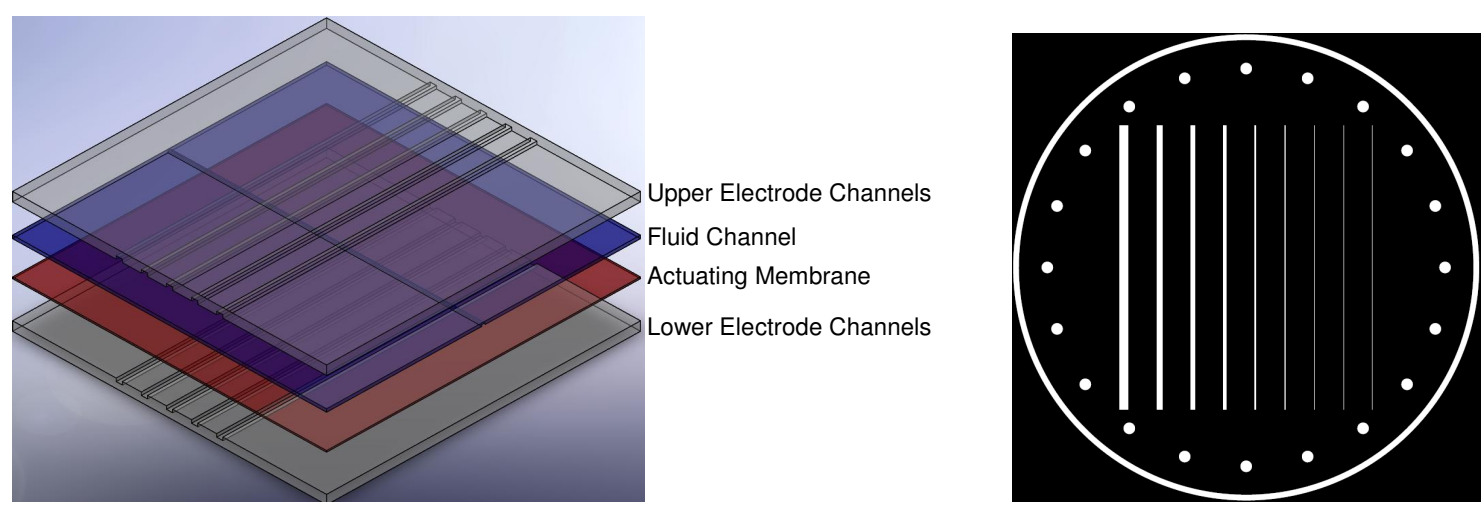

Figure 5.1 Left: Computer model of Concept II design. Right: Exposure mask for channel layer master molds.

The concept device consists of four layers. The upper and lower layers contain channels filled with silver liquid. These channels border a third set of channels which contain the fluid directed by the valve. Using the new plasma bonding recipe developed for the RIE allowed for far more uniform bonding across large PDMS surfaces, which encouraged another attempt at multiple valves in a single channel block. Each channel layer contained nine channels measuring 1500 $\mu \mathrm{m}, 1000 \mu \mathrm{m}, 750 \mu \mathrm{m}, 500 \mu \mathrm{m}, 250 \mu \mathrm{m}, 200 \mu \mathrm{m}, 150 \mu \mathrm{m}, 100 \mu \mathrm{m}$, and $50 \mu \mathrm{m}$. The $20 \mu \mathrm{m}$ tall fluid channels assemble at a $90^{\circ}$ angle to the electrode channels, creating 81 combinations of electrode and channel size. The $100 \mu \mathrm{m}$ electrode channel height attempted to alleviate expected flow complications during the electrode filling process. 


\subsection{Fabrication Procedure}

\section{Upper and Lower Electrode Channels}

1. Pour $30 \mathrm{ml}$ uncured PDMS into petri dish containing electrode channel master mold

2. Degas PDMS in vacuum chamber

3. Cure PDMS in oven at $75 \stackrel{\circ}{\circ}$ for 45 minutes

4. Cut channel block free from master mold

\section{Actuating membrane}

1. Spin coat PDMS onto bare wafer at 2,000 RPM for 30 seconds

2. Cure PDMS in oven at $75 \stackrel{\circ}{\mathrm{C}}$ for 45 minutes

\section{Fluid Channel Layer}

1. Spin coat PDMS onto bare wafer at 2,000 RPM for 30 seconds

2. Cure PDMS in oven at $75 \stackrel{\circ}{\circ}$ for 45 minutes

\section{Device Assembly}

1. Punch input/output ports into the upper and lower electrode channels.

2. Plasma bond lower electrode channel and fluid channel layer

3. Punch input/output ports into fluid channel layer

4. Plasma bond fluid channel layer and actuating membrane

5. Align and plasma bond actuating membrane and upper channel layer

6. Fill electrode channels with silver colloid

\subsection{Fabrication Complications and Solutions}

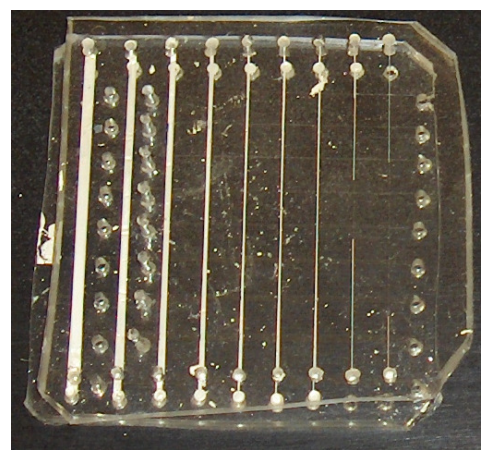

Figure 5.2 Completed silver liquid electrode prototype.

The resulting device failed to actuate. Channels larger than 500 microns wide underwent channel collapse during initial fabrication attempts. Increasing the actuation membrane curing temperature 
to $135{ }^{\circ} \mathrm{C}$ introduced a small pre-stress into the film and prevented channel collapse in channels $1,000 \mu \mathrm{m}$ or smaller during later fabrication attempts.

The thinnest channels proved impossible fill with silver liquid, as the pressure would rupture the membrane at the input port before fully filling the channel. This revealed the primary design flaw in the chip: the vertical stacking of the input ports left a $40 \mu \mathrm{m}$ thin film to support the pressures required to fill the channel. The high pressures required to fill the electrode channels tended to rupture the membranes sealing the valves and the input ports, leading to extreme cases of leaking within the chip.

Interfacing with the chip proved impossible due to an unforeseen interaction of the silver liquid with the PDMS channels. Ideally, the PDMS channels should have prevented the liquid drying inside the channel itself. However, the permeability of PDMS to air and solvent allowed the silver colloid to dry and settle. The polymer solution formed a solid insulative casing around the conductive element, rather than remaining in a liquid state. While ideal for repairing broken traces, the insulative layer prevented most attempts at interfacing with the traces. The act of breaking through the casing to interface electrically typically ruptured a membrane in the device and caused further leaking. 


\section{Chapter 6: Aluminum on PDMS Sputtering}

The failure of the liquid silver electrodes required a new method of electrode fabrication. Previous attempts to sputter aluminum directly onto PDMS substrates had limited success. Aluminum sputtering would intermittently leave a black scorched surface at points. In some cases scorching covered the entire substrate.

RF sputtering processes used on PDMS substrates behave oddly due to the insulative nature of the material. Unlike a conductive or semiconductive material, the charge imparted by the incoming metal ions cannot dissipate through the material. When these charges build up at various points on the surface of the substrate, they deflect incoming metal ions away. This property could cause the point scorching seen in previous sputtering attempts.

Plasma treatment just prior to sputtering uniformly polarizes the surface. A uniformly polarized surface presents a more stable electrical profile than uncharged PDMS. In theory, the polar surface could significantly reduce the generation of point charges and allow for more uniform metal coatings. Unfortunately, the treated surface relaxes quickly at normal room conditions, normally within 10-15 minutes. The standard operating procedure indicates that pumping down the sputtering chamber for less than 30-40 minutes can generate contaminated films with poor conductive properties.

\subsection{Experiment I - Plasma Treated Surfaces}

This experiment tested the effect of plasma treatment on the quality of aluminum sputtered traces on PDMS surfaces.

\section{Experiment I Procedure}

1. Use a Kapton tape shadowmask to cover all PDMS areas except a $2 \mathrm{~mm}$ wide line.

2. Treat substrate in RIE at $20 \mathrm{~W}$ and $308 \mathrm{mT}$ for 15 seconds

3. Place substrate in sputtering chamber and pump down for 45 minutes

4. Sputter aluminum at $8 \mathrm{mT}$ and $60 \mathrm{~W}$ for 10 minutes.

5. Remove Kapton mask from substrate. 


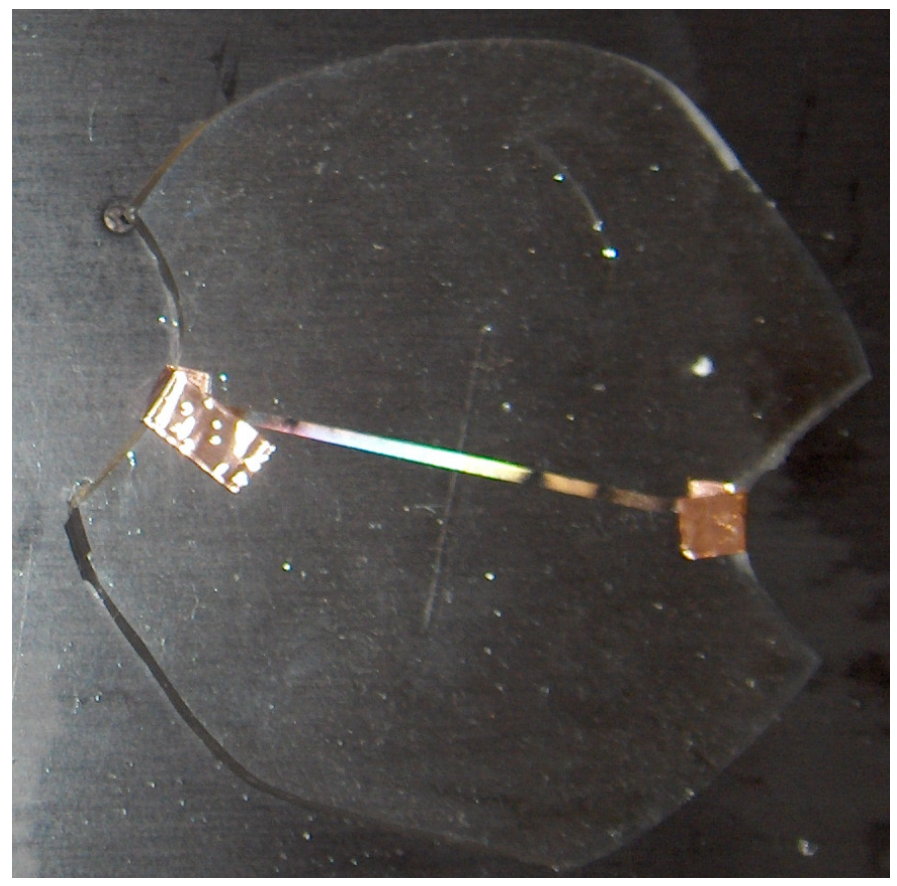

Figure 6.1 Point scorching along the $2 \mathrm{~mm}$ trace effectively creates an open line, destroying the circuit.

The procedure failed to produce a high quality film in the first 3 substrates. The length of the sputtering pump down exceeded the window of time in which PDMS surfaces relax from their plasma treated state. The next set of substrates used the same procedure with a reduced pump down time of 10 minutes. This sample had less point scorching, and all scorching originated at points of contact with the kapton tape mask. A third set of substrates with no kapton tape mask using the reduced pump down time created a high quality aluminum film with no point scorching.

\subsection{Experiment II - Decontamination of chamber}

While Experiment I resulted in an effective recipe, the first few substrates coated each run would experience extreme scorching across the entire surface of the substrate. After these sacrificial substrates, the recipe worked until the sputter coating chamber remained unused for more than a 2-3 hours. The fact that the defective coatings disappeared as the chamber spent more time under vacuum indicated chamber or target contamination as a likely cause of the problem. The reduced pump down time needed to begin sputtering prior to substrate relaxation likely failed to fully decontaminate the chamber.

Comparing the first sets of substrates sputtered on two days supported this theory. Each set of substrates consisted of 3 glass slides with a PDMS thin film spun and cured on each surface. The first day's substrates followed the recipe created in Experiment I. As expected, the three substrates experienced extreme scorching. The second day followed a modified procedure. 


\section{Experiment II Procedure}

1. Pump down sputtering chamber for 1 hour.

2. Sputter aluminum onto empty chamber for 5 minutes.

3. Use a Kapton tape shadowmask to cover all PDMS areas except a $2 \mathrm{~mm}$ wide line.

4. Treat substrate in RIE at $20 \mathrm{~W}$ and $308 \mathrm{mT}$ for 15 seconds

5. Place substrate in sputtering chamber and pump down for 45 minutes

6. Sputter aluminum at $8 \mathrm{mT}$ and $60 \mathrm{~W}$ for 10 minutes.

7. Remove Kapton mask from substrate.

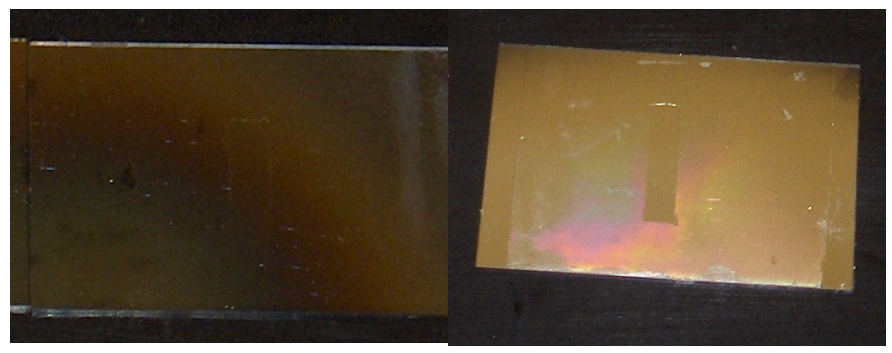

Figure 6.2 Left: Sputtering in a contaminated chamber results in extreme scorching. Right: Decontamination of the sputter chamber results in pristine films.

Pristine aluminum films resulted from this process. Measurement with the 4-point probe resulted in an experimental sheet resistance of $1.62 \pm 0.65 \Omega$. Calculation of the resistivity of the film requires film thickness data. Accurate thickness measurements of the film remain impossible as removing the aluminum film to obtain a step height causes the surface to become too soft for accurate profilometry measurements.

\subsection{Discussion and Conclusions}

Combating the scorching effect when sputtering onto PDMS requires both plasma treatment to polarize the surface and sputtering chamber decontamination. Simply plasma treating without decontaminating the chamber usually results in a hard, scorched film covering the surface of the substrate. While the film possesses extremely poor conductive properties, the film does not appear to grow after initial covering of the surface. The thin, hard nature of the film makes it ideal for coating substrates for profilometer measurements.

Eliminating the scorching problem altogether required decontaminate the chamber and sputtering target prior to sputtering plasma treated PDMS. Pumping down the chamber for 1 hour and sputtering aluminum on the empty chamber for 5 minutes sufficiently decontaminated the chamber environment and resulted in pristine aluminum films. 


\section{Chapter 7: Concept III - Aluminum Electrodes}

\subsection{Iteration I - Flexible backbone}

The ability to sputter aluminum directly onto PDMS allowed new electrode fabrication techniques. Concept III used aluminum electrodes to deliver an actuation voltage to the valve. The design began as a revision of the original gold electrode concept, modified slightly to account for differences in process techniques.

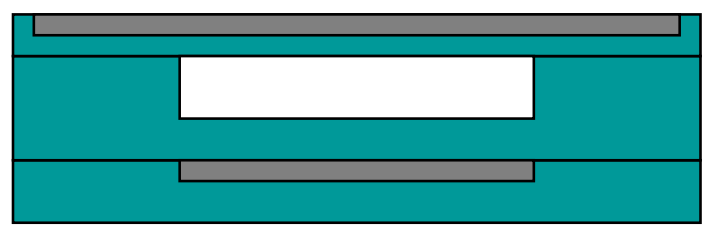

Upper Electrode Layer

Channel Layer

Lower Electrode Layer

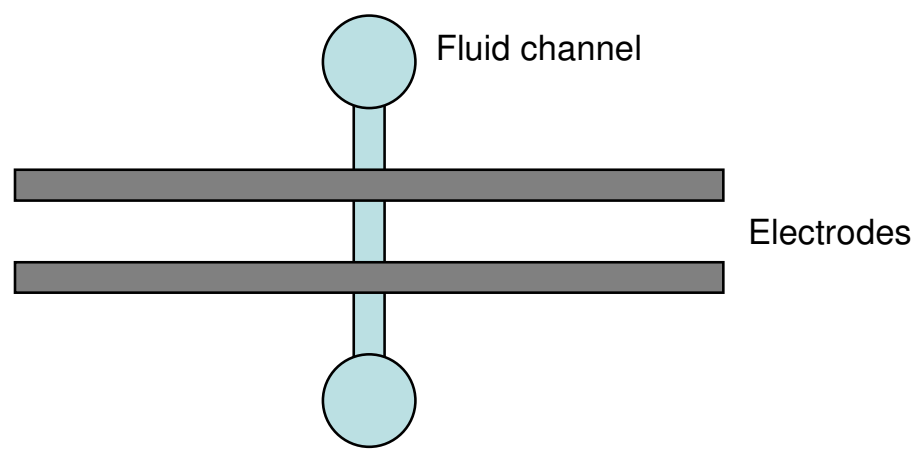

Figure 7.1 Above: Grey areas depict placement of aluminum electrodes in each layer. Below: Top down view of basic device design.

Complexity in previous devices resulted in small point failures destroying entire chips. Concept III simplified the design by placing only one fluid channel on each device and creating only two valves on that channel. The simplified design reduced areas where leakage problems found in earlier devices could occur.

The lower electrode layer contains both the lower electrode sets and the backbone that stabilizes the entire channel structure during handling. The first PDMS based lower electrode layers retained the Concept I target thickness of $0.4 \mathrm{~cm}$. A kapton mask allowed sputtering of $1 \mathrm{~mm}$ wide aluminum traces onto the PDMS. The $30 \mu \mathrm{m}$ thick channel layer contained a $20 \mu \mathrm{m}$ deep, 750 $\mu \mathrm{m}$ wide channel imprinted into the film. The upper layer cured at an elevated temperature to induce thermal contraction prior to bonding to prevent channel collapse. Once the upper layer bonded to the assembly, the entire upper layer received a 15 minute aluminum sputter coat treatment. 


\subsection{Iteration I Fabrication}

Lower Electrode Layer

1. Pour $0.4 \mathrm{~cm}$ thick block of PDMS

2. Pump down sputtering chamber for 1 hour

3. Cure in oven for 45 minutes at $75^{\circ} \mathrm{C}$

4. Apply Kapton tape shadowmask

5. Plasma treat masked PDMS block at $308 \mathrm{mT}$ and $20 \mathrm{~W}$ for 15 seconds

6. Immediately transfer PDMS to sputtering and pump down for 8 minutes

7. Pre-sputter aluminum for 1 minute at $60 \mathrm{~W}$ and $9 \mathrm{mT}$

8. Open sputter shield and sputter aluminum for 15 minutes at $60 \mathrm{~W}$ and $9 \mathrm{mT}$

9. Remove block from sputter chamber and remove Kapton shadowmask

\section{Channel Layer}

1. Spin $30 \mu \mathrm{m}$ thin film of PDMS over $20 \mu \mathrm{m}$ tall SU-8 master channel mold

2. Cure in oven for 45 minutes at $75^{\circ} \mathrm{C}$

\section{Upper Electrode Layer}

1. Spin $20 \mu \mathrm{m}$ thin film of PDMS over silicon wafer treated with JerseyCote

2. Cure in oven for 20 minutes at $135^{\circ} \mathrm{C}$

\section{Assembly}

1. Plasma treat Lower Electrode Layer and Channel Layer for $15 \mathrm{sec}$ at $308 \mathrm{mT}$ and $20 \mathrm{~W}$

2. Place treated surfaces in contact and cure in oven at $75 \stackrel{\circ}{\circ}$

3. Plasma treat Channel Layer and Upper Electrode Layer for $15 \mathrm{sec}$ at $308 \mathrm{mT}$ and $20 \mathrm{~W}$

4. Place treated surfaces in contact and cure in oven at $75^{\circ} \mathrm{C}$

5. Plasma treat Upper Electrode Layer for 15 seconds at $308 \mathrm{mT}$ and $20 \mathrm{~W}$

6. Immediately transfer device to sputtering chamber

7. Pump down chamber for 8 minutes

8. Pre-sputter aluminum for 1 minute at $60 \mathrm{~W}$ and $9 \mathrm{mT}$

9. Open sputter shield and sputter aluminum for 15 minutes at $60 \mathrm{~W}$ and $9 \mathrm{mT}$

10. Remove device from chamber

11. Isolate and attach copper conductive tape to lower traces and upper ground layer 


\subsection{Fabrication Complications}

The Lower Electrode Layer could not supply adequate support during handling to function as a backbone. Severe cracking of the aluminum films occurred during handling. The lower electrodes rarely survived plasma bonding of the channel layer without extreme cracking problems. The Kapton tape method of electrode patterning also resulted in mild point scorching even after plasma treatment and decontamination.

The lack of easy access to the lower electrodes resulted in damage during electrical packaging procedures. The upper PDMS layers of the electrostatic valve blocked electrical contact to the lower electrodes. The act of removing the upper layers covering the electrode contacts typically destroyed the trace itself in the process.

Accessing these electrodes required selective removal of the PDMS layers above the electrodes without high mechanical strain or excessive scraping of the substrate below. Selectively bonding the upper layers allowed selective removal of specific sections of PDMS away from the active area of the device.

Generating a selective bond required a small modification of the plasma treatment process. A Kapton tape shadowmask applied to the surface prior to placing the substrates into the plasma treatment chamber prevented treatment of the masked areas. The untreated areas did not bond during the oven curing step and created a removable flap. This flap protected the interface area of the lower traces during processing, prior to removal during the packaging stage of assembly.

\subsection{Iteration II - Glass Backbone}

Switching to 2 in $\times 3$ in glass substrates for the Lower Electrode Layer allowed access to known lithography and wet etching methods, and provided the necessary support to prevent severe cracking of the lower aluminum electrode. Changing the backbone substrate to glass slides required significant alteration of the fabrication process of PDMS layers.

Any SU-8 structures built onto a silicon master mold typically create minor irregularities in the surface of any PDMS thin films spun onto the mold. Unlike a PDMS block, a glass substrate does not compress during the contact phase of bonding. Irregularities in the surface of a PDMS layer can generate air pockets when attempting to bond to a bare glass substrate and cause widespread bond failure.

Additionally, release layers used in prior fabrication methods proved ineffective. Once a glass substrate bonded to a thin film coated onto a silicon wafer, the rigidity and brittleness of the substrates prevented the peeling action required to release the PDMS layer. Attempting to separate the PDMS layer invariably resulted in the shattering of either the glass slide or the SU-8 
master mold. The glass slide backbone required more flexible mold substrates. Plastic petri dishes used as spincoating platforms by other projects allowed a semistable platform for spincoating with a degree of flexibility, allowing the necessary peeling action during release.

Plastic mold substrates present a key design problem. Wet chemistries used to develop SU-8 photoresist severely damage plastic substrates. This forces the use of other molding methods and leads to problems creating channel layers with proper tolerances.

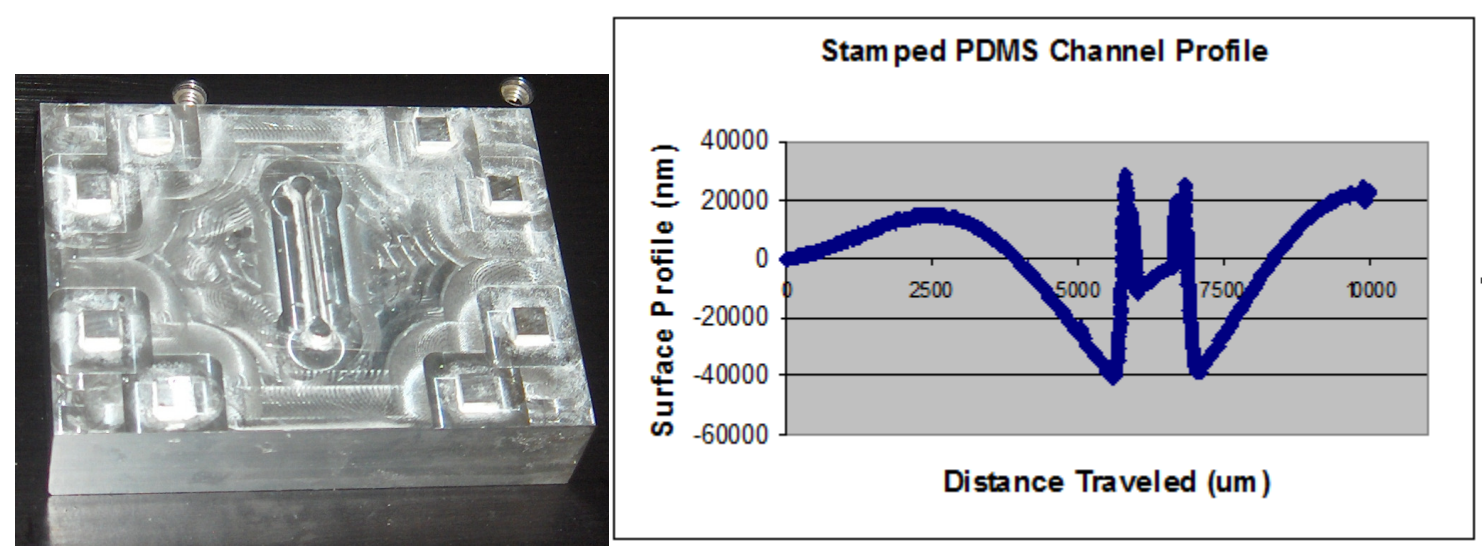

Figure 7.2 Left: Channel stamp block. Right: Profilometer scan along stamped PDMS surface postcure.

The first prototype device used an aluminum stamp to create a channel pattern in the channel layer after spincoating directly onto a plasma treated glass backbone. The stamp procedure failed when the PDMS climbed the sides of the aluminum stamp during the curing step, and created several thousand micron surface irregularities around the channel, preventing later bonding steps.

The second prototype device used a Kapton tape mold. The Kapton tape mold consisted of a piece of Kapton tape placed on the center of the plastic substrate and cut to the shape of the channel. Spincoating PDMS onto the mold resulted in irregular surfaces that caused air pockets during bonding and ultimately resulted in failure of the bond. The solution to the air pocket problem combined the curing and plasma bonding procedures.

\subsection{Iteration II Fabrication}

\section{Glass Backbone}

1. Clean glass slide in Piranha Etchant for 10 minutes at $70^{\circ} \mathrm{C}$

2. Place glass slide in sputter chamber and pump down for 45 minutes

3. Sputter aluminum onto glass slide at $60 \mathrm{~W}$ and $9 \mathrm{mT}$ for 15 minutes

4. Spin coat positive photoresist onto glass slides

5. Bake photoresist at $90{ }^{\circ} \mathrm{C}$ for 1 minute 
6. Expose photoresist using aluminum trace mask for 16 seconds

7. Develop photoresist in CD-26 for 10 minutes

8. Etch Aluminum using Transene Type A etchant

9. Strip photoresist using acetone

10. Etch any aluminum on sides of glass slide using Transene Type A etchant

\section{Channel Layer}

1. Apply Kapton tape to plastic substrate

2. Cut Kapton tape to shape of channel structure

3. Pour liquid PDMS onto center of petri dish

4. Apply Kapton tape selective bonding mask to glass backbone

5. Plasma treat glass backbone in RIE at $308 \mathrm{mT}$ and $20 \mathrm{~W}$ for 30 seconds

6. Remove Kapton tape selective bonding mask

7. Press treated glass backbone into liquid PDMS

8. Place plastic substrate onto aluminum block in oven and place aluminum weight onto glass backbone

9. Cure in oven at $75^{\circ} \mathrm{C}$ for 45 minutes

10. Peel plastic substrate away from glass backbone and channel layer.

\section{Actuating Membrane}

1. Spin coat PDMS onto plastic substrate at 2000 RPM for 30 seconds

2. Cure in oven at $135^{\circ} \mathrm{C}$ for 15 minutes

\section{Assembly}

1. Plasma treat channel layer and actuating membrane in RIE at $308 \mathrm{mT}$ and $20 \mathrm{~W}$ for 15 seconds

2. Place channel layer and actuating membrane in contact and cure in oven at $75{ }^{\circ} \mathrm{C}$ for 30 minutes

3. Pump down sputtering chamber for 1 hour

4. Peel valve assembly from plastic substrate

5. Plasma treat valve assembly in RIE at $308 \mathrm{mT}$ and $20 \mathrm{~W}$ for 15 seconds

6. Transfer valve assembly to sputtering chamber

7. Pump down chamber for 8 minutes

8. Pre-sputter aluminum for 1 minute at $60 \mathrm{~W}$ and $9 \mathrm{mT}$

9. Open sputter shield and sputter aluminum for 15 minutes at $60 \mathrm{~W}$ and $9 \mathrm{mT}$

10. Remove device from chamber

11. Remove selectively bonded flaps to expose lower traces

12. Isolate and attach copper conductive tape to lower traces and upper ground layer 


\subsection{Fabrication Complications}

Iteration II marked the first successful fabrication of a testable prototype. All electrodes tested positive for connection macroscopically and no fluid leaks occurred. One of the valves collapsed during bonding, leaving one valve intact. This valve did not actuate upon application of a driving voltage.

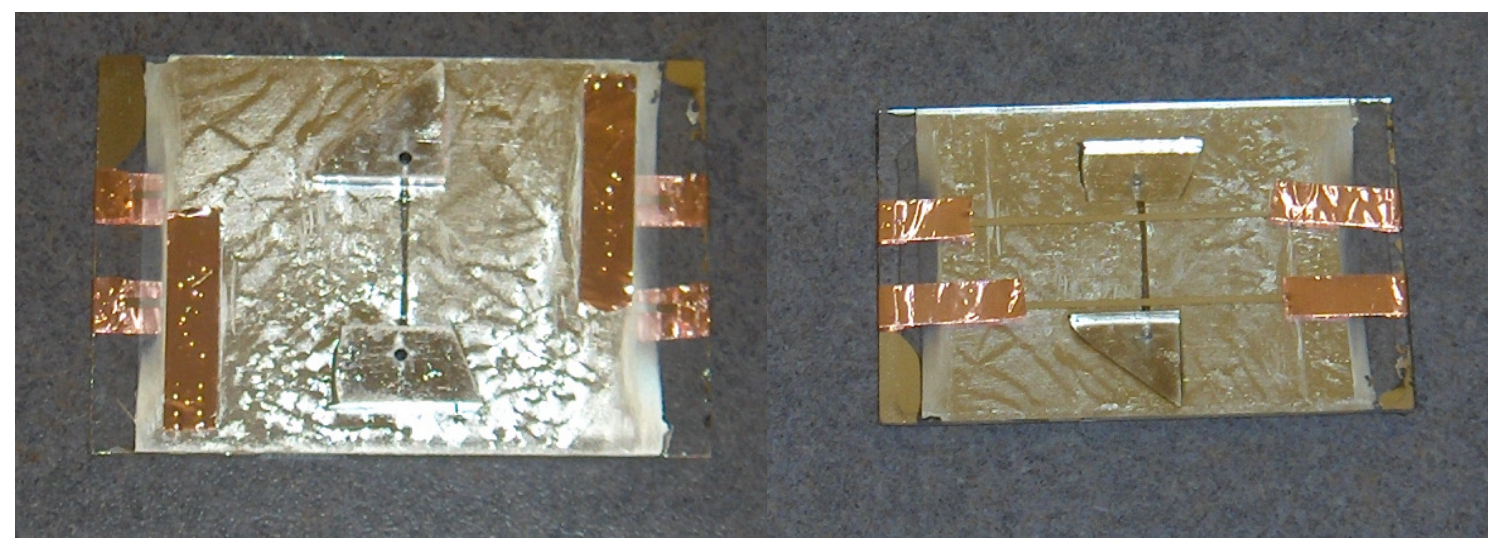

Figure 7.3 Left: Top of Prototype Electrostatic Valve. Right: Underside of prototype electrostatic Valve.

Investigating the valve failure revealed two possible causes. Localized cracking at the channel edges prevented a driving voltage from applying across the channel properly. The cracking formed bands of isolated aluminum over the valve itself. These isolated bands received no driving voltage and effectively cut the upper electrode from the circuit entirely.

Additionally, a cross section of two channel layers from devices that never completed fabrication revealed extreme inconsistency in the distance between the bottom of the channel layer and the lower electrodes. Without precise control of the distance between the electrodes the driving voltage became extremely difficult to predict.

\subsection{Conclusions}

Aluminum sputtering can successfully create a conductive layer on PDMS. However, the fragility of the sputtered layers causes major fabrication complications. Attempts to stabilize the aluminum with rigid substrates met with moderate success. Aluminum layers on PDMS with direct rigid substrate support eliminate widespread cracking problems but retain localized cracking problems near surface irregularities.

Patterning of aluminum on PDMS presents severe difficulties. Shadowmasking PDMS layers engendered scorching during the sputtering steps, and wet etching processes performed on PDMS supported aluminum led to damage to the PDMS layer under the aluminum. Generating a patterned aluminum trace on PDMS layers proved impossible to achieve. Layer-wide sputtering 
of aluminum showed promise as effective ground layers in areas away from device geometry. The severe cracking which occurs near device geometries on layers below the aluminum layer made aluminum a non-ideal material for electrode fabrication. 


\section{Chapter 8: Aluminum Cracking Investigation}

The capacitive circuit used in the valve required no actual flow of electrons across the valve itself. The charge sourced on one side of a crack should have induced a voltage bias and transferred at least some voltage to the valve itself, yet applying a voltage across the valve generated no detectable movement of the concept III valve. Assessing the viability of aluminum electrodes required explaining why the capacitive nature of the circuit did not overcome the cracking problem.

Four major handling conditions can cause cracking. Light contact with the surface from fingers or other objects causes slight localized cracking. Removal of Kapton masks used to pattern aluminum on PDMS creates regions of cracking around the interfaces during lift off. The act of lifting off a PDMS layer supporting an aluminum film creates extreme cracking. Finally, any bending of the substrate creates significant cracking across the entire aluminum film.

\subsection{Light Contact}

Micrographs of light contact on an aluminum on PDMS film revealed cracking in radial patterns emanating from the point of contact.

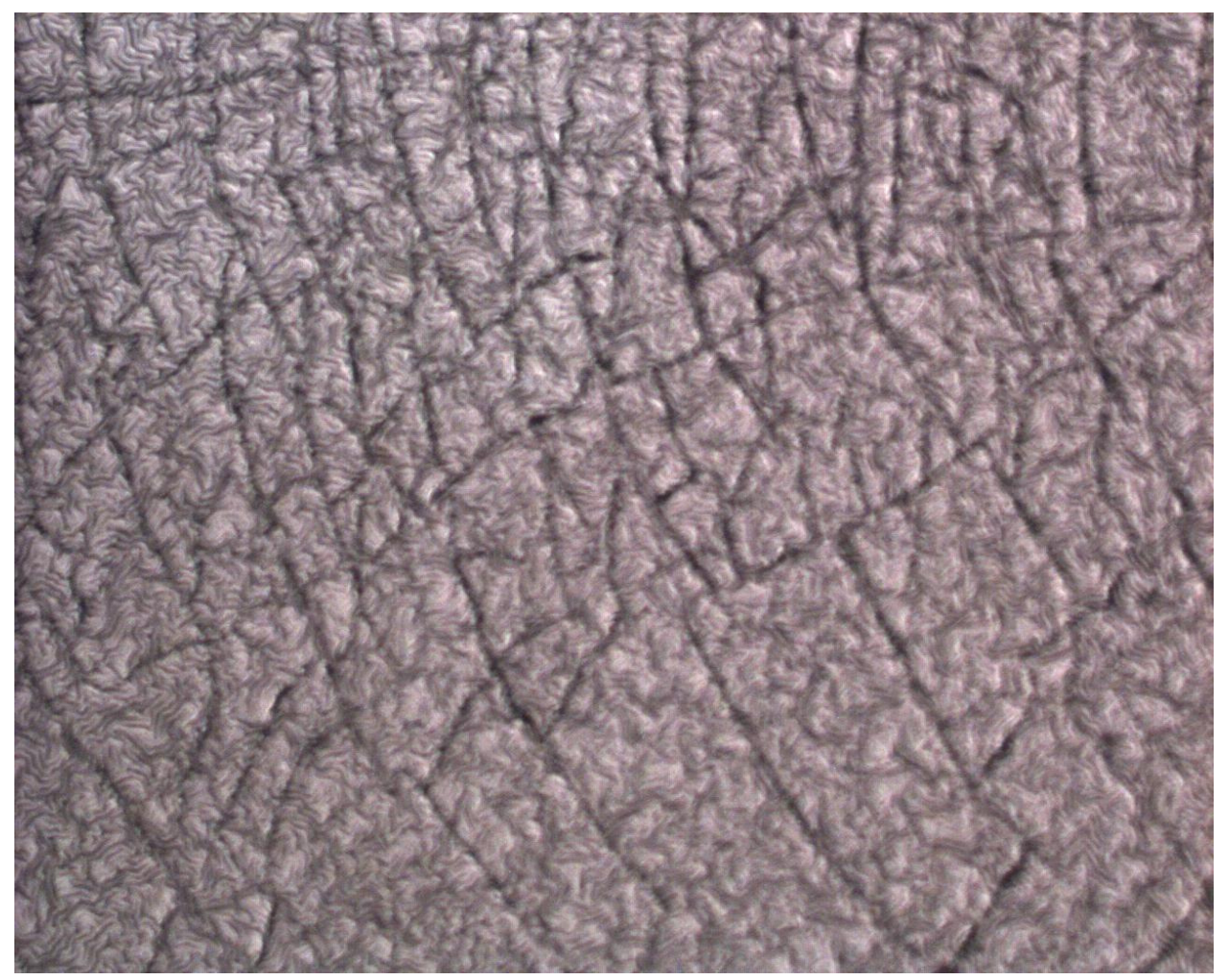

Figure 8.1 Light contact cracking creates a radial pattern of closed aluminum cells. 
Most notably, the patterns created small cells of aluminum isolated by cracks on all sides. These cracks emanate from the central point of pressure from any given light contact. The pressure applied by light contact deforms the thin film of PDMS supporting the aluminum film locally. The PDMS elongates at a rate the aluminum cannot match and cracking occurs quickly due to the fragility of aluminum films only hundreds of nanometers thick.

\subsection{Kapton Removal}

Removing Kapton tape from aluminum sputtered onto a PDMS surface left extreme surface cracking in any film under the tape as well as fringe effects at the edge of the tape-substrate interface.

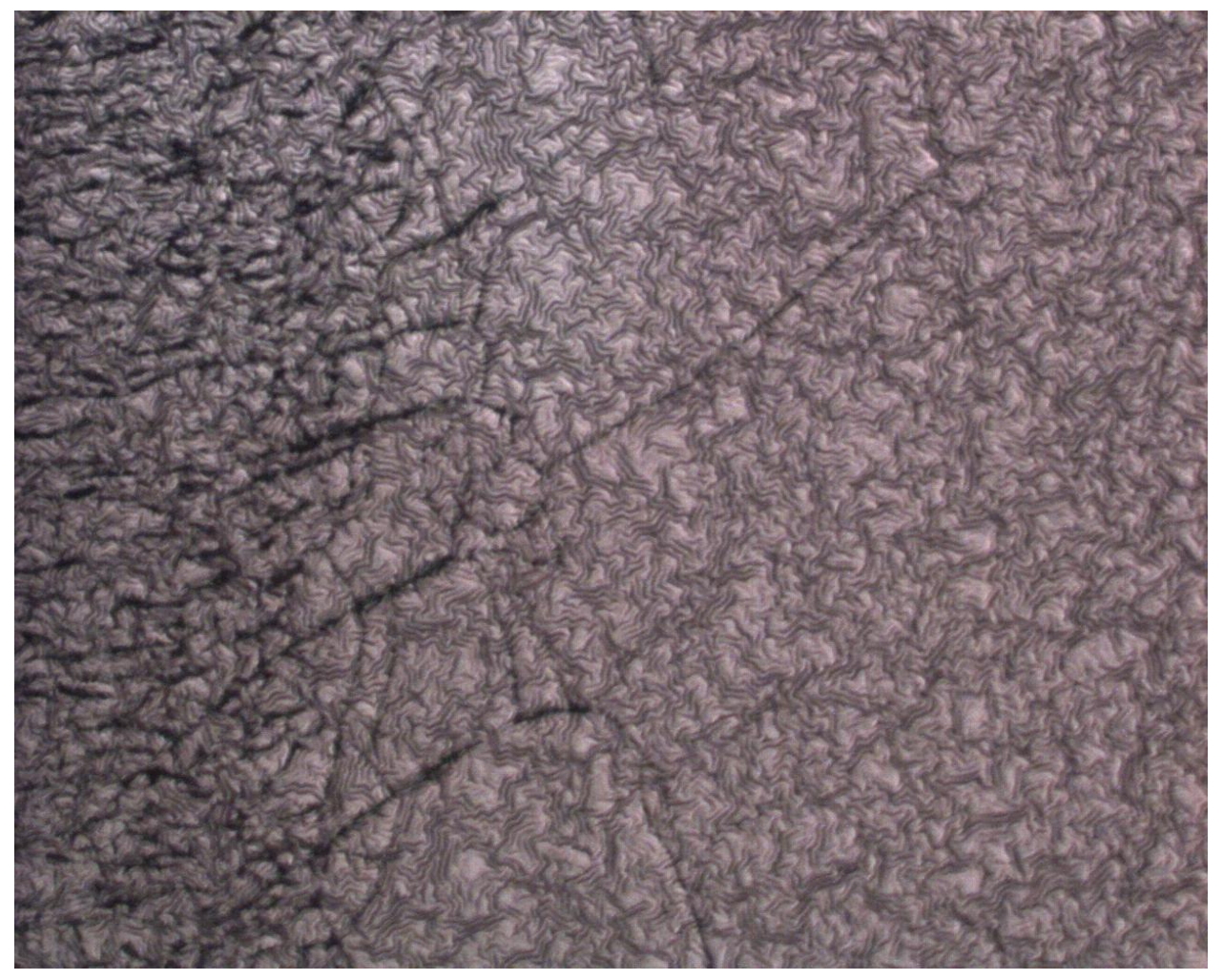

Figure 8.2 Removing Kapton tape from an aluminum film causes extreme surface damage and formation of isolated cells.

When the Kapton tape peels from the surface, the PDMS film deforms under the stress well beyond the ductility of the brittle aluminum film. The area near the tape also deforms to a lesser degree, generating the fringe cracking. Eliminating the scorching problem caused by Kapton tape masking remains pointless because this edge cracking alone will isolate large sections of any aluminum trace patterned with Kapton masking. 


\subsection{Lift Off and Peeling}

Removing an aluminum coated PDMS layer from a glass substrate effectively replicated the mechanical stress imposed on the aluminum film during a standard lift off via peeling procedure.

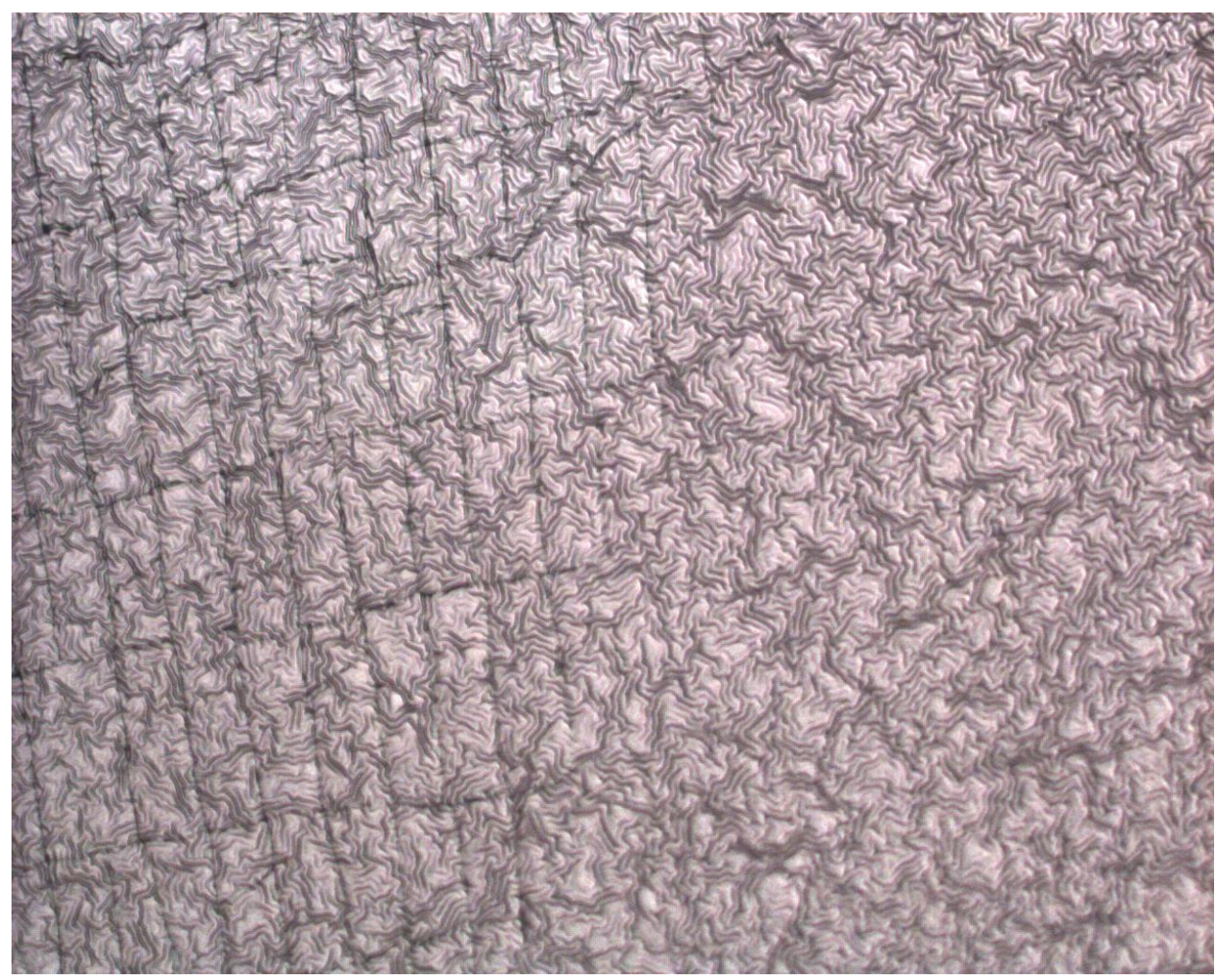

Figure 8.3 Peeling of aluminum coated thin films creates isolated rectangular cells.

Micrographs of the peeling interface show rectangular cracking patterns. This rectangular pattern appears to only appear under lift off conditions, as tension occurs in multiple directions at the same time. These rectangular cracks create obvious electrical isolation between cells.

\subsection{Substrate Bending}

Bending any substrate with an aluminum film creates fairly large damage to the film itself. Examining samples from the first iteration of concept III reveals heavy cracking from bending during handling. 


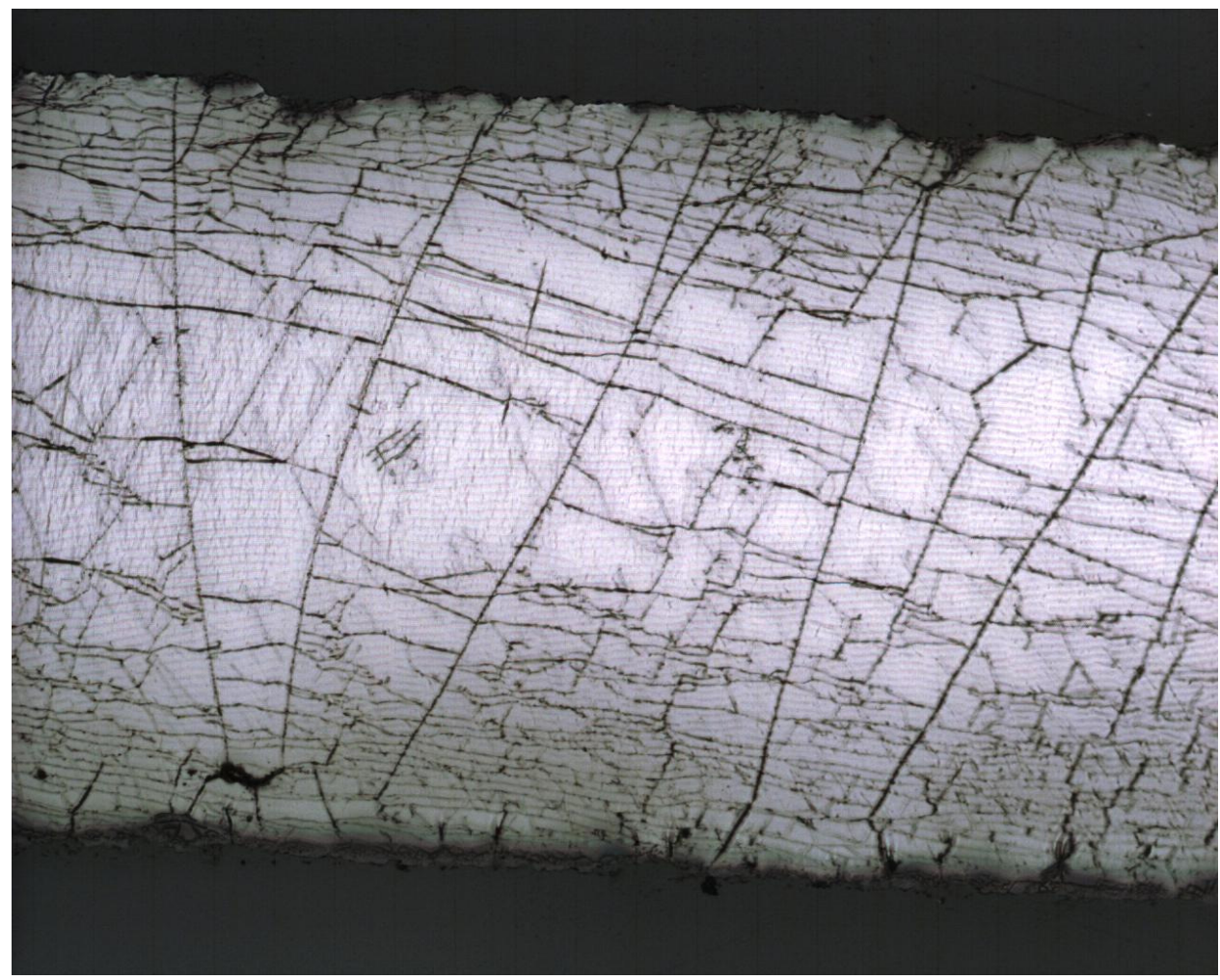

Figure 8.4 Aluminum traces on PDMS blocks show extreme cracking after even minor amounts of handling.

While the kapton tape used to mask these samples causes most of the cracking at the sides of the traces, the central cracking only appears after a few hours of handling. PDMS substrates prove far too flexible to support aluminum traces without additional structural support in the form of a glass backbone.

\subsection{Conclusions}

The degree of cracking occurring under normal handling procedures creates isolated cells in the aluminum traces. These cells demonstrated an interesting difference between the theoretical diagram for the valve circuit and reality. In the original circuit theory, induced voltage across cracks should have generated enough charge at the active portion of the circuit to actuate the device. This theory hinged on relatively minor cracking. The sheer amount of cracking created a device with not just one or two cracks to induce a voltage across, but thousands. Even if the source voltage induced a voltage in all of these cells, predicting the relative voltage to the ground electrode remains impossible. Additionally, while sections of each cell became charged, that charge never resulted in a net increase or decrease in electrons present in the bulk electrode. The force generated by the charge never increased enough to actuate the valve. 
The presence of these isolated cells within the active area of a valve removed any chance of the valve functioning. The brittleness of aluminum films revealed during handling makes aluminum non-viable as an electrode material. A viable electrostatic valve required another material switch. 


\section{Chapter 9: Findings and Future Work}

\subsection{Summary of Findings}

Fabrication of an electrostatic switch ultimately remained unsuccessful. The additional materials and development time required to take the next logical step after the failure of concept III exceeded the remaining resources and allotted timeframe of this master's thesis. While the concept designs failed to result in a working device, each concept has added significantly to the knowledge base of the Cal Poly Clean Room.

Concept I attempted to reproduce the results of a paper describing the process to transfer gold films into PDMS. Exact replication of the conditions of the experiment required unavailable equipment, and all attempts to modify the process to fit clean room technology failed. Moving to oxide release films showed that reducing adhesion prior to patterning caused failure of the metallic film during wet etching.

Plasma bonding experiments allowed the creation of recipes for bonding of PDMS blocks to both glass and other PDMS blocks. PDMS to glass bonding requires 30 seconds of exposure to $\mathrm{O}_{2}$ plasma at $308 \mathrm{mT}$ and $20 \mathrm{~W}$, while PDMS to PDMS bonding requires 15 seconds of exposure to $\mathrm{O}_{2}$ plasma at $308 \mathrm{mT}$ and $20 \mathrm{~W}$.

Concept II complications led to a thermal pre-stress technique used to prevent channel collapse during fabrication steps. Increasing the cure temperature of the thin membrane covering a channel to $135 \stackrel{\circ}{\circ}$ induces enough pre-stress to prevent channel collapse in channels up to 1,000 $\mu \mathrm{m}$ wide.

Concept II showed improper placement of interface points in multilayer devices can lead to severe leaking. Active device areas require structures with a sufficient bonding area to separate active device areas or device pressures can cause bonds or layer integrity to fail.

Concept II also showed the air and solvent permeability of PDMS can allow solvent based suspensions to dry within channels.

Aluminum sputtering experiments showed plasma exposure of PDMS helped eliminate the effect of point charges during sputtering, which eventually led to a process to sputter both pristine aluminum films and hard glassy coatings. The aluminum coatings proved extremely useful for taking profilometer readings of PDMS step heights.

Concept III, Iteration I showed the need for access flaps to reach lower electrode interfaces. Shadow masking during plasma treatment allowed the creation of partial bonds that in turn allowed easy removal of trace coverings. 
Iteration I also showed PDMS backbones failed to protect against cracking in the metallic thin film. Iteration II used glass backbone layers help eliminate most cracking of aluminum films sputtered onto PDMS. However, critical areas near device features still exhibited severe cracking. Fabricating valves directly onto a glass backbone created greater fabrication complications than the change resolved.

Stamping fabrication techniques showed that small surface irregularities can prevent full contact of two treated surfaces during plasma bonding. Lack of contact between surfaces prevents bonding in those areas and invariably leads to leaking and failure.

The aluminum cracking investigation showed cracking of aluminum films resulted in small, isolated cells which prevented net charge buildup over the active valve area. PDMS layers simply cannot provide the mechanical support necessary to eliminate cellular cracking of brittle metallic films in device critical areas.

\subsection{Recommendations for Future Work}

The results of concepts I, II, and III lead to the following guidelines for future PDMS-based electrostatic work at Cal Poly.

1. Use as few layers as possible. Each layer added to a device compounds the chances of failure exponentially, as all previous points of failure can fail during bonding as well as all new critical areas on the added layer.

2. Reduce layer complexity to a minimum. Leave at least $10,000 \mu \mathrm{m}$ between fluid channels whenever possible to avoid leaking.

3. Avoid using brittle materials in PDMS based devices. The lack of rigid support from PDMS during fabrication and handling processes render brittle materials non-ideal for PDMS based microfluidic devices.

4. Use large valve structures. Valves less than $500 \mu \mathrm{m}$ wide quickly increase the amount of voltage required to close the valve exponentially.

Options for further research into valve electrodes include Dow Corning® DA 6254 and electrically conductive polymers. DA 6254 contains over $60 \%$ silver particles suspended in PDMS with adhesive components. Adding high amounts of silver to Sylgard 184 could create similar compounds without the adhesive properties. Electrically conductive polymers also represent a possible avenue of research into flexible, micropatternable, electrodes.

Metallization with more ductile metals such as copper could also solve the cracking problems. Electronics applications have used copper coated polymers as flexible connectors for many years. 
Further research into thermal pre-stressing of PDMS membranes could greatly increase the maximum channel width available to PDMS devices created in the Cal Poly Clean Room.

Selective bonding presents an opportunity for a highly valuable research project. Selective bonding techniques could provide further expansion of the tools available in soft lithography processes and further open multilayer design possibilities.

Aluminum sputtered PDMS layers allow creation of proper PDMS spin speed curves. These curves could remove a significant amount of trial and error from the fabrication process and heavily increase device yield in all PDMS based microfluidics at Cal Poly. 


\section{References}

[1] S. K. Sia and G. M. Whitesides. Microfluidic Devices Fabricated in Poly(dimethylsiloxane) for Biological Studies. Electrophoresis. 2003. 24: 3563-3576.

[2] J. M. K. Ng, I. Gitlin, A. D. Stroock, and G. M. Whitesides. Components for Integrated Poly(dimethylsiloxane) Microfluidic Systems. Electrophoresis. 2002. 23: 3461-3473.

[3] Z. Yang, S. Matsumoto, H. Goto, M. Matsumoto, and R. Maeda. Ultrasonic Micromixer for Microfluidic Systems. Sensors and Actuators A. 2001. 93. 266-272.

[4] T. N. T. Nguyen, M-C. Kim, J-S. Park, and N-E. Lee. An Effective Passive Microfluidic Mixer Utilizing Chaotic Advection. Sensors and Actuators B. 2008.

[5] V. Studer, G. Hang, A. Pandolfi, M. Ortiz, W. F. Anderson and S. R. Quake. Scaling Properties of a Low-Actuation Pressure Microfluidic Valve. Journal of Applied Physics. 2004. 95: 393-398.

[6] M. Yamada and M. Seki. Nanoliter-Sized Liquid Dispenser Array for Multiple Biochemical Analysis in Microfluidic Devices. Anal. Chem. 2004. 76: 895-899.

[7] O. C. Jeong, and S. Konishi. Fabrication and Drive Test Pneumatic PDMS Micro Pump. Sensors and Actuators A. 2007. 135: 849-856.

[8] W. H. Grover, A. M. Skelly, C. N. Liu, E.T. Lagally and R. A. Mathies. Monolithic Membrane Valves and Diaphragm Pumps for Practical Large-Scale Integration into Glass Microfluidic Devices. Sensors and Actuators B. 2003. 89: 315-323.

[9] J. Wang. Portable Electrochemical Systems. Trends in Analytical Chemistry. 2002. 21:226232,

[10] J. C. McDonald and G. M. Whitesides. Poly(dimethylsiloxane) as a Material for Fabricating Microfluidic Devices. Accounts of Chemical Research. 2002. 35: 491-499.

[11] S. R. Quake and A. Scherer. From Micro- to Nanofabrication with Soft Materials. Science. 2000. 290: 1536-1539.

[12]. Thuillier and C. K. Malek. Development of a Low Cost Hybrid Si/PDMS Multi-layered Pneumatic Microvalve. Microsyst. Technol. 2005. 12: 180-185.

[13] See www.coleparmer.com

[14] M. A. Unger, H-P. Chou, T. Thorsen, A. Scherer, and S. R. Quake. Monolithic Microfabricated Valves and Pumps by Multilayer Soft Lithography. Science. 2000. 288: 113-116.

[15] See www.dowcorning.com 
[16] J.C. Lötters, W. Olthuis, P. H. Veltink, and P. Bergveld. The Mechanical Properties of the Rubber Elastic Polymer Polydimethylsiloxane for Sensor Applications. J. Micromech. Microeng. 1997. 7:145-147.

[17] H. Yang, Q. T. Nguyen, Y. Ding, Y. Long, and Z. Ping. Investigation of poly(dimethyl siloxane) (PDMS)-solvent interactions by DSC. Journal of Membrane Science. 2000. 164: 37-43

[18] see www.filmetrics.com

[19] S. Middlemann and A. Hochberg. Process Engineering Analysis in Semiconductor Device Fabrication. McGraw-Hill College. 1993. 313-320

[20] See www.microchem.com

[21] C. Liu. Foundations of MEMS. Pearson Education. 2006.

[22] E. P. Kartalov, A. Scherer, S. R. Quake, C. R. Taylor and W. F. Anderson. Experimentally Validated Quantitative Linear Model for the Device Physics of Elastomeric Microfluidic Valves. Journal of Applied Physics. 2007. 101

[23] K. J. Lee, K. A. Fosser, and R. G. Nuzzo. Fabrication of Stable Metallic Patterns Embedded in Poly(dimethylsiloxane) and Model Applications in Non-Planar Electronic and Lab-on-a-Chip Device Patterning. Advanced Functional Materials. 2005. 15: 557-566. 


\section{Appendix A: PDMS Thin Film Data}
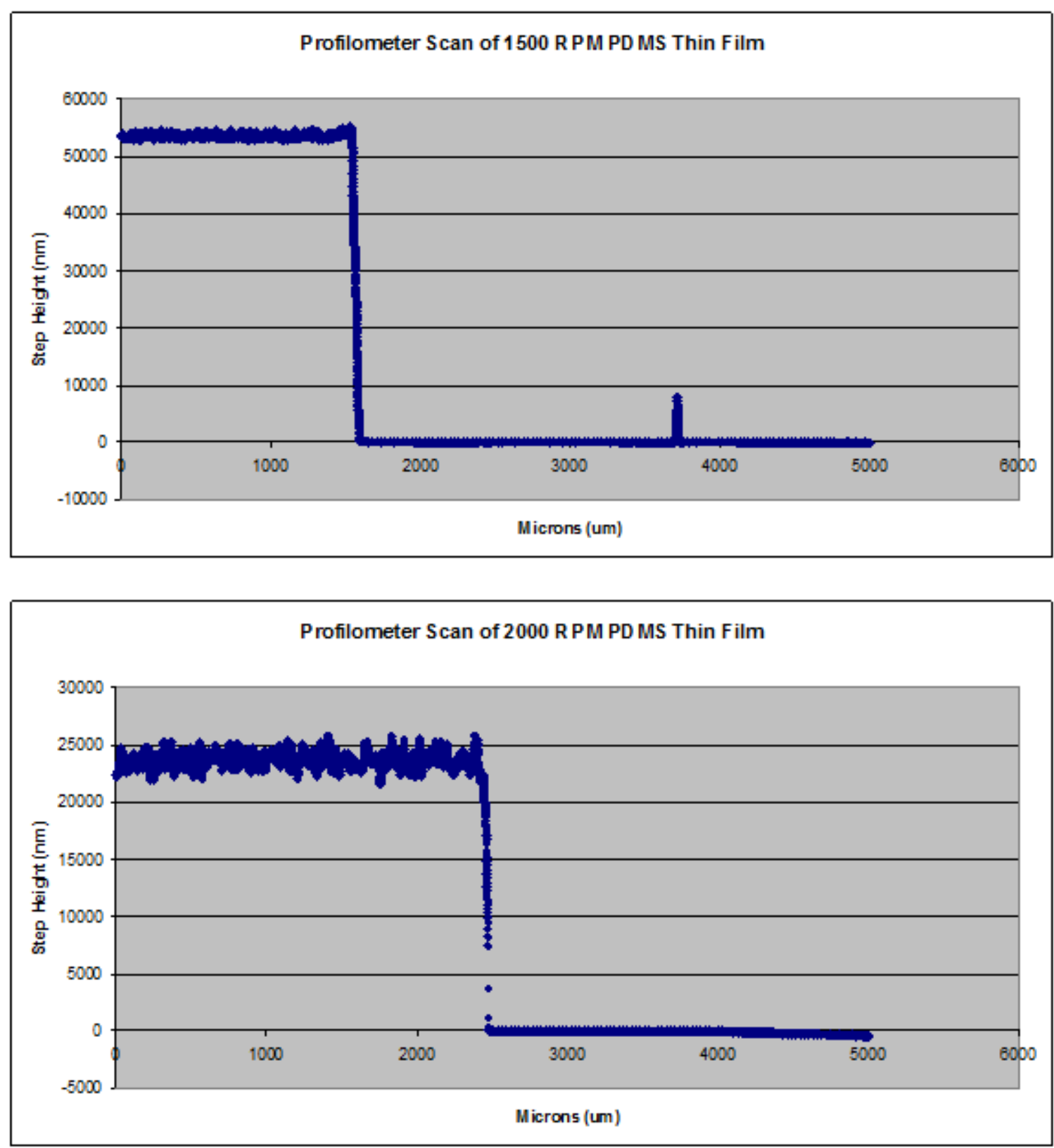

Figure A.1 Step height of 1500 RPM and 2000 RPM Thin Films after metallization and cross sectioning. 


\section{Appendix B: Selective Bonding Photos}

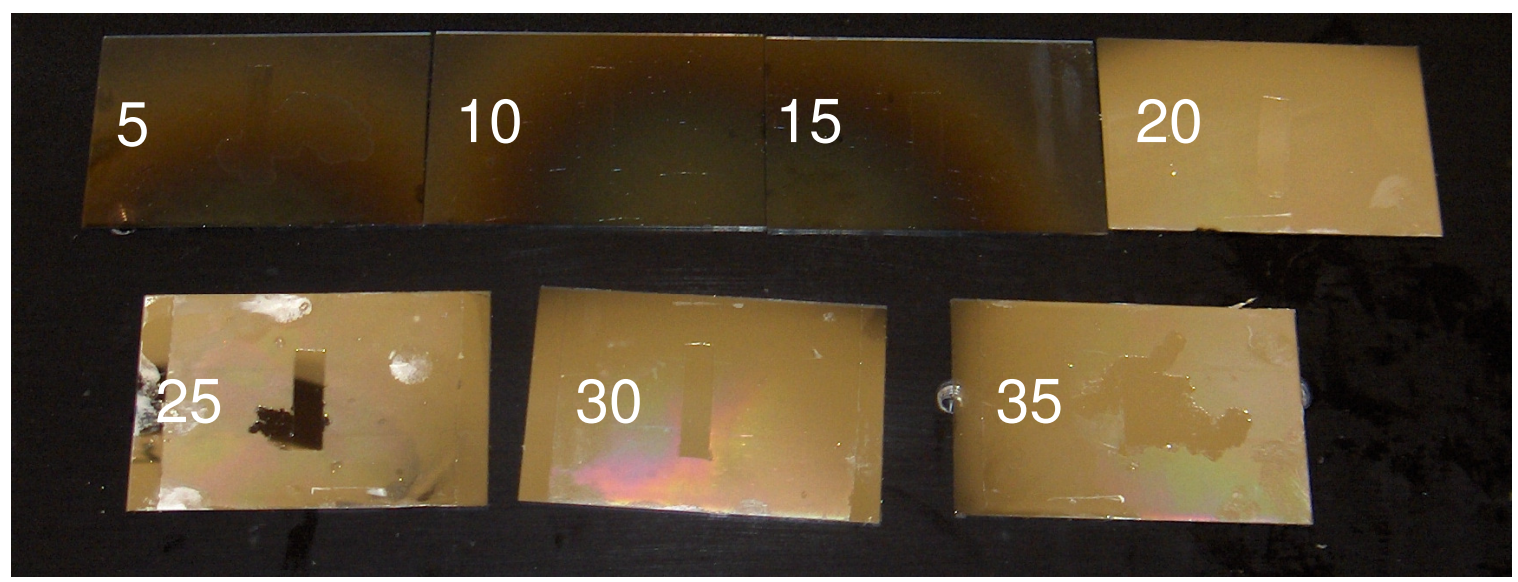

Figure B.1 Selective Bonding test results optimal bonding at 30 second exposure. The first three samples also show good examples of substrate wide sputter scorching.

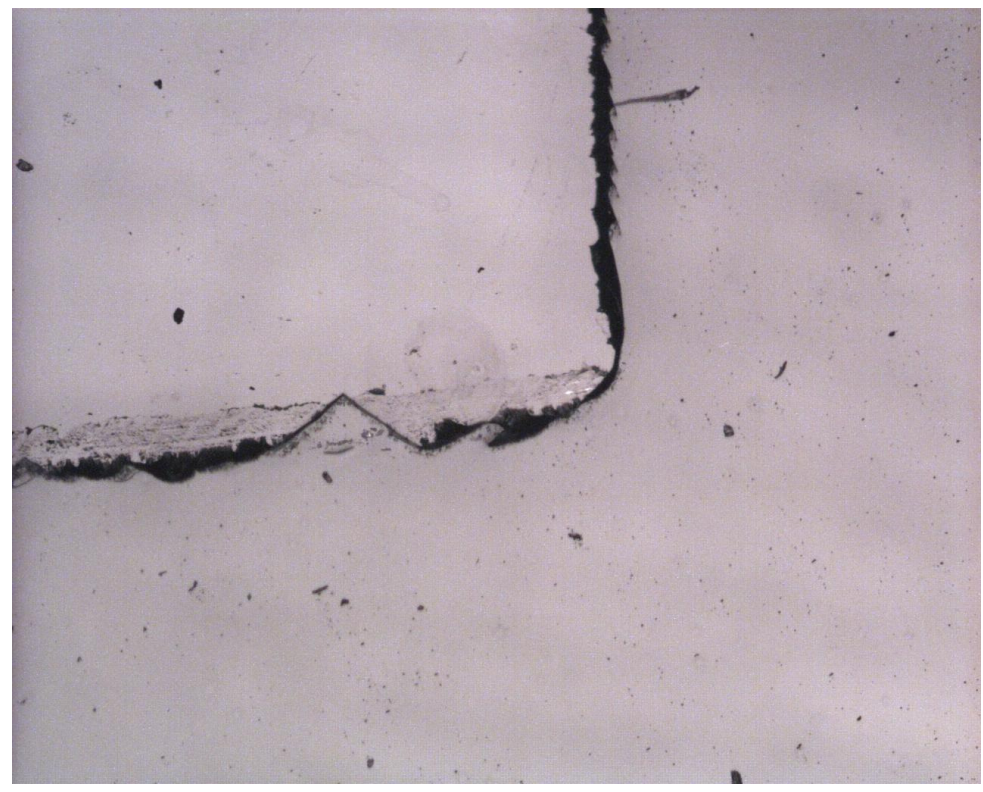

Figure B.2 Micrographs of the selective bonding interface after tearing the flap away show a remarkably clean break. 